\title{
Identification of coexisting dynamics in boundary layer flows through proper orthogonal decomposition with weighting matrices
}

\author{
Matteo Dellacasagrande (D) Dario Barsi • Patrizia Bagnerini - Davide Lengani • \\ Daniele Simoni
}

Received: 9 September 2019/Accepted: 20 April 2021/Published online: 7 May 2021

(C) The Author(s) 2021

\begin{abstract}
A different version of the classic proper orthogonal decomposition (POD) procedure introducing spatial and temporal weighting matrices is proposed. Furthermore, a newly defined non-Euclidean (NE) inner product that retain similarities with the POD is introduced in the paper. The aim is to emphasize fluctuation events localized in spatiotemporal regions with low kinetic energy magnitude, which are not highlighted by the classic POD. The different variants proposed in this work are applied to numerical and experimental data, highlighting analogies and differences with respect to the classic and other normalized variants of POD available in the literature. The numerical test case provides a noisefree environment of the strongly organized vortex shedding behind a cylinder. Conversely, experimental data describing transitional boundary layers are used to test the capability of the procedures in strongly not uniform flows. By-pass and separated flow transition processes developing with high free-stream disturbances have been considered. In both cases streaky structures are expected to interact with other vortical structures (i.e. free-stream vortices in the by-pass case and Kelvin-Helmholtz rolls in the separated type) that carry a significant different amount of energy. Modes
\end{abstract}

M. Dellacasagrande $(\bowtie) \cdot$ D. Barsi .

P. Bagnerini - D. Lengani · D. Simoni

DIME - Università di Genova, Via Montallegro 1,

I-16145 Genova, Italy

e-mail: matteo.dellacasagrande@edu.unige.it obtained by the non-Euclidean POD (NE-POD) procedure (where weighted projections are considered) are shown to better extract low energy events sparse in time and space with respect to modes extracted by other variants. Moreover, NE-POD modes are further decomposed as a combination of Fourier transforms of the related temporal coefficients and the normalized data ensemble to isolate the frequency content of each mode.

Keywords POD - Frequency content - Weighting matrices $\cdot$ Boundary layers-free stream interaction

\section{Introduction}

Modal decomposition techniques represent nowadays fundamental tools for data analysis and construction of reduced order models (ROMs) of complex systems. In this context, POD represents an energy-ranked decomposition technique, looking for the basis maximizing the projection of the entire ensemble of data ([24]). In its classic formulation, POD solves the eigenvalue problem for the positive-defined spatial correlation tensor, or, in the successive snapshot POD of Sirovich [39], for the computationally more convenient temporal cross-correlation matrix. Recent applications of POD to Particle Image Velocimetry (PIV) data clearly highlight the ability of POD in tracking vortices shed in the wake of cylinders ([31]), blunt bodies ([34]) as 
well as in the case of boundary layer separation ([19]). The POD modes and related temporal coefficients constitute two orthonormal basis for their own vector space, and can be consequently adopted for the projection of each other quantity of interest. Indeed, following the extended POD procedure proposed by Borée [3], the spatial POD modes of a given field can be computed as projection on the temporal coefficients obtained by solving the POD problem in a different spatial domain, or with different quantities used for the definition of the POD kernel. Among others, Antoranz et al. [1] used extended POD to inspect the evolution of coherent motions due to thermal gradients in turbulent pipe flow, highlighting the quota of velocity fluctuations correlating with the temperature ones (see also the works of $[8,14])$. The orthogonality condition of the POD modes and the related temporal coefficients gives also the attractive advantage of splitting Reynolds normal and shear stresses ([24]), as well as the contributions to the production of TKE of the flow and the corresponding dissipation, as shown in recent authors' publications ([20, 37] and [22]). However, as stated in Sieber et al. [35], POD is not useful when the dominant structures appear at multiple frequencies or spatial wavelengths. The procedure proposed by Sieber et al. [35] basically consists of filtering the convolution matrix and successively applying the POD to the filtered kernel. The filter width, accounting for the overall scale of the dominant structures, switches the procedure from a classic POD to a descrete Fourier transform (DFT). The cost to be paid is the loss of orthogonality of the spatial modes and a higher dispersion of the POD spectrum. A similar filtering procedure has been introduced into the works of Iudiciani et al. [16] and Bourgeois et al. [4] to develop a generalized phase-averaging procedure based on the POD. In Bourgeois et al. [4] the snapshot matrix was filtered by means of the convolution with a Gaussian window to detect the base flow variation characterizing the transient vortex shedding behind a cylinder, that appears captured by the most energetic POD modes of the filtered kernel. The successive decomposition of the residual allows maximizing the energy captured by the POD of the residual crosscorrelation matrix, thus maximizing the energy captured by the phase-averaged field. In the more recent work of Mendez et al. [28] a different approach named multi-scale POD was introduced to overcome the limitation of the classic POD when this latter is applied in the case of flow with multiple frequencies. The authors apply a filter on the convolution matrix based on multi-resolution analysis. POD therefore provides statistics for each frequency bands considered, which can be combined afterwords for the characterization of the overall fluctuations embedded within the flow. Differently from the spectral POD method of Sieber et al. [35], the stationarity of the data set is not forced since the variance of each diagonal of the convolution matrix is not modified.

In case of phase-dependent flows, like in internal combustion engine or turbomachinery applications (see e.g., [7, 11, 21, 33, 42]), data snapshots are characterized by significant energy variation (between the phases), and events occurring at low energetic phases are barely captured by the classic POD methods. The phase-invariant POD proposed in Fogleman et al. [10], and adopted in engine applications in Voisine et al. [42], normalizes each snapshot by its own norm prior to construct the cross-correlation matrix. This provides the attractive advantage of highlighting low energy events sparse in phase, or more generally in time (i.e. each time/phase is treated in the same way, being normalized). This normalization process can be generalized introducing a weighting matrix into the scalar product providing the projection of the data ensemble on the optimum basis obtained by the POD method, similar to the problem formulated in Sarmast et al. [32] to account for not uniform spatial grids.

From this perspective, the classic POD procedure and other variants known in the literature are reformulated in the present work in terms of weighting matrices, providing a more general formulation. The adaptation of particular choices for these matrices allows highlighting turbulent events embedded in spatio-temporal regions with low kinetic energy. Based on the approach of Sarmast et al. [32], we first consider a spatial normalization of the snapshot matrix to make turbulent events occurring at different positions more comparable in terms of energy. Then, we introduce a non-Euclidean scalar product into the classic POD procedure to consider a not uniform distribution of the flow kinetic energy in both space and time. This is the main feature characterizing the present work with respect to other variants available in the literature. It is pointed out here that the coexistence of correlating events in spatial regions with different energy of fluctuations could be exploited also by 
means of the aforementioned extended POD method. However, this would require to split the available data set beforehand, based on the previous knowledge of the regions where correlating events may occur. Additionally, extended POD modes provide the statistical representation of such dynamics separately. The aim of the present method is to provide evidence of the simultaneous occurrence of velocity fluctuations with different energy content, without introducing a priori any split of the particular data set at hand. The application of the procedures here examined to different test cases show that the introduction of a nonEuclidean inner product allows keeping the benefits of previous methods and further highlighting flow oscillations sparse in time.

The procedures are applied to both numerical and experimental data. The first concerns with literature data obtained from direct numerical simulation (DNS) describing the vortex shedding behind a circular cylinder (see the book of Kutz et al. [18] for the reference to the link for downloading and Brunton et al. [5] for the POD analysis of these data). This test case actually describes a strongly periodic flow pattern, with structures that are equally distributed in the different snapshots, but appearing in different spatial positions. Once applied to flow fields with significant energy variation on both time and space, the POD variants presented provide different modal representation of turbulent events occurring in spatiotemporal regions at different energy.

Two experimental test cases have been also considered in this work. A by-pass like transition process forced by homogeneous free-stream turbulence provides a test case where free-stream vortices are energetically decoupled by the more energetic boundary layer streaks (see $[17,45]$ ). The second data set concerns a pressure induced laminar separation bubble undergoing laminar-turbulent transition. Due to the elevated homogeneous free-stream turbulence characterizing also this condition, streaky structures are expected to influence the shear layer dynamics, prior to the formation of the most energetic KelvinHelmholtz $(\mathrm{K}-\mathrm{H})$ rolls at the bubble maximum displacement position (see [15, 25, 27, 38, 44]). These experimental cases are discussed in order to emphasize the capability of the weighted scalar product to highlight spatio-temporal regions with low kinetic energy with respect to a symmetric normalization of the snapshot matrix. Moreover, the NE-POD modes are further decomposed as a combination of Fourier transforms of temporal coefficients and velocity maps, thus better highlighting flow regions with different energy and frequency content. This is something similar to what applied by Glauser and George [12], Arndt et al. [2] and Liu et al. [23] in homogeneous spatial directions.

The paper is organized as follows: in Sect. 2 the mathematical formulation providing a rational generalization of the different methods is presented. In Sect. 3 the different procedures are applied to the DNS data. In Sect. 4.1 the by-pass transition case will be discussed, while in Sect. 4.2 the separation-induced transition process is presented. Finally, concluding remarks on the advantages of the present techniques are synthesized in Sect. 6.

\section{Mathematical framework}

\subsection{Classic snapshot POD}

For all the methods presented here, we consider $U$ to be the snapshot matrix containing a set of observations of the velocity field in the spatio-temporal domain. Let $\mathbf{x}=(x, y)$ be the spatial coordinates and $u_{i}=u\left(\mathbf{x}, t_{i}\right)$ be the velocity field at the point $\mathbf{x}$ and time $t_{i}$ for $i=1, \cdots, N$. The experimental values are obtained in space with a uniform mesh $\mathbf{x}_{j}=j \Delta \mathbf{x}$ for $j=1, \ldots, M$ and discrete time interval with constant step size $\Delta t$. The vectors $u_{i}$ have then dimension $M$ and form the columns of $U$. We instead denote the rows of $U$ by $u^{j}$, having dimension $N$. Since in both DNS and PIV applications the number of spatial points $(M)$ is typically much larger than the number of snapshots collected $(N)$, the snapshot POD method of Sirovich [39] is here introduced for reference. This problem is equivalent to find the coefficient vector $v_{1}$ that maximizes the projection of the row vectors of $U$, and the POD algorithm corresponds to solve the following constrained maximization problem:

$$
\max _{\left\|v_{1}\right\|^{2}=1}\left\|U v_{1}\right\|^{2}=\max _{\left\|v_{1}\right\|^{2}=1}\left(U v_{1}, U v_{1}\right)
$$

where $(\cdot, \cdot)$ is the Euclidean inner product. Denoting by ${ }^{T}$ the matrix transpose, the problem (1) can be rewritten as 


$$
\max _{\left\|v_{1}\right\|^{2}=1} v_{1}^{T} U^{T} U v_{1}=\max _{v_{1}^{T} v_{1}=1} v_{1}^{T} C v_{1}
$$

where $C$ is the correlation matrix

$$
C=U^{T} U
$$

The constrained problem (2) can be solved using Lagrange multipliers to transform it in an unconstrained problem. Let $\mathscr{L}\left(v_{1}, \lambda_{1}\right)=v_{1}^{T} C v_{1}+\lambda_{1}(1-$ $\left.v_{1}^{T} v_{1}\right)$ be the Lagrangian functional with $\lambda_{1} \in \mathfrak{R}$ the Lagrange multiplier, the solution of (2) satisfies $\nabla_{v_{1}} \mathscr{L}\left(v_{1}, \lambda_{1}\right)=C v_{1}-\lambda_{1} v_{1}=0$, i.e.

$$
C v_{1}=\lambda_{1} v_{1}
$$

This shows that $v_{1}$ is an eigenvector of the correlation matrix $C$ and $\lambda_{1}$ is the corresponding eigenvalue. The first POD mode can be then easily obtained by projection:

$$
\phi_{1}=U v_{1}
$$

The second component $\phi_{2}$ can be computed in analogue way, by computing $v_{2}$ as eigenvector of $C$ with eigenvalue $\lambda_{2}$ and so on, obtaining a set of $N$ orthogonal eigenvectors and sorted eigenvalues due to the symmetry and positive definition of $C$. Since it is convenient to work with an orthonormal basis, the vectors $\phi_{i}$ for $i=1, \ldots, N$ are rescaled to have unitary norm, i.e. the relation (5) is replaced by

$$
\phi_{i}=U v_{i} \lambda_{i}^{-1 / 2}, \quad i=1, \ldots, N
$$

which is equivalent to compute a $M \times N$ matrix $\Phi$ defined as

$$
\Phi=U V Z
$$

where $V$ is the matrix with the eigenvectors $v_{i}$ on the columns and $Z$ the diagonal matrix having $\left(N \lambda_{i}\right)^{-1 / 2}$ on its diagonal. The column vectors of $\Phi$ composed by $\phi_{i}$ for $i=1, \ldots, N$ are the POD modes.

\subsection{Time invariant POD (TI-POD)}

Fogleman et al. [10] introduced a variant of the classic POD with the aim of making events sparse in time equally probable. In this paper, the authors refer to the procedure as a phase-invariant method, being it applied to phase-dependent flows. Here it is referred as Time-Invariant POD (TI-POD), being applied with the aim of normalizing each snapshot in not phasedependent applications. The procedure basically consists in applying the POD algorithm not to the original matrix $U$, but to the normalized snapshot matrix $\tilde{U}$ defined as

$$
\tilde{U}=U W_{T}
$$

where $W_{T}$ is a diagonal $N \times N$ matrix of weights chosen in order to make the columns of $U$ unitary. This is obtained dividing each column by the Euclidean norm of $u_{i}$ or in other words dividing by the spatial root mean square (rms) of velocity fluctuations of each observation:

$$
W_{T}=\left(\begin{array}{cccc}
\frac{1}{\left\|u_{1}\right\|} & & & 0 \\
& \frac{1}{\left\|u_{2}\right\|} & & \\
& & \ddots & \\
0 & & & \frac{1}{\left\|u_{N}\right\|}
\end{array}\right)
$$

Then, the maximization problem (2) for the TI-POD becomes:

$$
\max _{\left\|v_{1}\right\|^{2}=1} v_{1}^{T} W_{T} U^{T} U W_{T} v_{1}=\max _{v_{1}^{T} v_{1}=1} v_{1}^{T} C v_{1}
$$

where the correlation matrix $C$ has in this case the expression:

$$
C=W_{T} U^{T} U W_{T}
$$

Note that the symmetry of the correlation matrix is preserved. Then, the POD modes are computed as

$$
\Phi=\tilde{U} V Z
$$

where as before $V$ is the matrix with the eigenvectors of $C$ and $Z$ the diagonal matrix having $\left(N \lambda_{i}\right)^{-1 / 2}$ on its diagonal. Note that Eq. (12) provides the modes for the normalized snapshot matrix $\tilde{U}$, according to the procedure described in the original work of Fogleman et al. [10]. However, the original field can be easily obtained as:

$$
U=\Phi Z^{-1} V^{T} W_{T}^{-1}
$$

Clearly, TI-POD modes correspond to the definition of the classic POD modes when $W_{T}=\llbracket$ (where $\llbracket$ is the identity matrix). 


\subsection{Space invariant POD (SI-POD)}

In spatially inhomogeneous flows, both phase or not phase dependent, velocity oscillations with different energy content can coexist and even interact (e.g. K-H rolls and free-stream vortices). With the aim of making these turbulent events more comparable in terms of energy, thus highlighting their coexistence by the modal decomposition of the snapshot matrix, a dual problem of the TI-POD is presented. Namely, another variant of the POD called Space Invariant POD (SI-POD) is proposed, where the normalization of time-traces instead of snapshots is considered. A similar approach was proposed by Sarmast et al. [32] to take into account a space discretization with a non uniform mesh, where the weights of the diagonal matrix modifying $U$ are the local cell volumes corresponding to each grid point. Indeed, when the spatial sampling of the correlation matrix defined in (3) is not uniform, the scalar product between two realizations has to be weighted to account for the different weights linked to the non-uniform spatial sampling, as described also in the previous work of Antoranz et al. [1].

In the procedure proposed here, the POD algorithm can be formulated considering a modified snapshot matrix $\tilde{U}$, defined as

$$
\tilde{U}=W_{S} U
$$

where $W_{S}$ is a diagonal $M \times M$ matrix of weights chosen in order to make the rows of $U$ unitary, obtained dividing each row of $U$ (denoted by $u^{j}$ for $j=1, \ldots, M)$ by its Euclidean norm:

$$
W_{S}=\left(\begin{array}{cccc}
\frac{1}{\left\|u^{1}\right\|} & & & 0 \\
& \frac{1}{\left\|u^{2}\right\|} & & \\
& & \ddots & \\
0 & & & \frac{1}{\left\|u^{M}\right\|}
\end{array}\right)
$$

Then, the maximization problem (2) becomes

$$
\max _{\left\|v_{1}\right\|^{2}=1} v_{1}^{T} U^{T} W_{S} W_{S} U v_{1}=\max _{v_{1}^{T} v_{1}=1} v_{1}^{T} C v_{1}
$$

where the correlation matrix $C$ has in this case the expression:

$$
C=U^{T} W_{S} W_{S} U=U^{T} W_{S}^{2} U
$$

with $W_{S}^{2}:=W_{S} W_{S}$. Note that $C$ is still symmetric, thus its eigenvetors are orthogonal. Following the TI-POD method previously described, the modes are computed by projection of the normalized snapshot matrix $\tilde{U}$

$$
\Phi=\tilde{U} V Z
$$

where as before $V$ is the matrix with the eigenvectors of $C$ and $Z$ the diagonal matrix having $\left(N \lambda_{i}\right)^{-1 / 2}$ on its diagonal. The original flow field is then reconstructed as:

$$
U=W_{S}^{-1} \Phi Z^{-1} V^{T}
$$

Another possible interpretation of the space invariant POD is to find $v_{1}$ that maximizes the weighted norm of the projection $U v_{1}$. From this perspective, the problem (16) is therefore equivalent to

$$
\begin{aligned}
& \max _{\left\|v_{1}\right\|^{2}=1}\left(U v_{1}, U v_{1}\right)_{W_{S}^{2}}=\max _{\left\|v_{1}\right\|^{2}=1} v_{1}^{T} U^{T} W_{S}^{2} U v_{1} \\
& \quad=\max _{v_{1}^{T} v_{1}=1} v_{1}^{T} C v_{1}
\end{aligned}
$$

where $C$ is defined as in (17). Also in this case $W_{S}=\rrbracket$ provides the classic formulation of Sirovich [39].

\subsection{Time-space invariant POD (TSI-POD)}

Starting from the TI-POD and SI-POD formulations presented in the previous sections, one could think to perform a Time-Space Invariant POD (TSI-POD) including the advantages of both temporal (column) and spatial (row) normalizations of the snapshot matrix, thus defining a new normalized snapshot matrix as:

$$
\tilde{U}=W_{S} U W_{T}
$$

In this context the correlation matrix would be defined as:

$$
C=W_{T} U^{T} W_{S} W_{S} U W_{T}
$$

with the TSI-POD modes being computed again by projection of the normalized field $\tilde{U}$ :

$$
\Phi=\tilde{U} V Z
$$


As for the previous variants presented, $V$ is the matrix with the eigenvectors of $C$ and $Z$ the diagonal matrix having $\left(N \lambda_{i}\right)^{-1 / 2}$ on its diagonal. However, it is easy to prove that the simultaneous normalization of both the rows and columns of $U$ cannot be achieved by the right and left multiplication with the weighting matrices $W_{S}$ and $W_{T}$.

\subsection{Non-Euclidean POD (NE-POD)}

A different approach is introduced in this section considering weighted products and norms in the optimization problem of the POD (Eq. 1). The introduction of the weighting matrices $W_{S}$ and $W_{T}$ into Eq. (1) makes the POD modes more sensitive to temporal normalization with respect to the SI-POD, still emphasizing oscillations occurring in spatial region with low kinetic energy, as it will be shown in Sect. 4. The modified version proposed here consists in solving the following maximization problem:

$$
\begin{aligned}
& \max _{\left\|v_{1}\right\|_{W_{T}}^{2}=1}\left\|\left(U v_{1}\right)_{W_{T}}\right\|_{W_{S}^{2}}^{2}=\max _{v_{1}^{T} W_{T} v_{1}=1}\left\|U W_{T} v_{1}\right\|_{W_{S}^{2}}^{2} \\
& =\max _{v_{1}^{T} W_{T} v_{1}=1} v_{1}^{T} W_{T} U^{T} W_{S}^{2} U W_{T} v_{1}=\max _{v_{1}^{T} W_{T} v_{1}=1} v_{1}^{T} W_{T} C v_{1}
\end{aligned}
$$

where $C$ is the weighted correlation matrix defined as

$$
C=U^{T} W_{S}^{2} U W_{T}
$$

Similar to what done with Eqs. (2), (25) can be solved using Lagrange multipliers. Let $\mathscr{L}\left(v_{1}, \lambda_{1}\right)=$ $v_{1}^{T} W_{T} C v_{1}+\lambda_{1}\left(1-v_{1}^{T} W_{T} v_{1}\right)$ be the Lagrangian functional, then the solution of (25) satisfies $\nabla_{v_{1}} \mathscr{L}\left(v_{1}, \lambda_{1}\right)=W_{T} C v_{1}-\lambda_{1} W_{T} v_{1}=0$, i.e.

$$
C v_{1}=\lambda_{1} v_{1}
$$

Since in this case the matrix $C$ is not symmetric, its eigenvectors are not orthogonal with respect to the Euclidean product, but they are orthogonal with respect to the weighted product $(\cdot, \cdot)_{W_{T}}$. At the same time, the resulted modes are orthogonal with respect to the weighted product $(\cdot, \cdot)_{W_{S}^{2}}$. The non-orthogonality of the NE-POD temporal coefficients implies that the contributions of the modes to the energy of the reconstructed field are not independent to each others and modal interaction exists. The NE-POD modes are obtained as

$$
\phi_{i}=\left(U, v_{i}\right)_{W_{T}} \lambda_{i}^{-1 / 2}=U W_{T} v_{i} \lambda_{i}^{-1 / 2}, \quad i=1, \ldots, N
$$

or in matrix notation as

$$
\Phi=U W_{T} V Z
$$

where as before $V$ is the matrix with the eigenvectors of $C$ and $Z$ the diagonal matrix having $\left(N \lambda_{i}\right)^{-1 / 2}$ on its diagonal. Note that the equation above is the same as (12), even if Eq. (29) provides the modes for the original snapshot matrix $U$, differently from (12). Hence, the original flow field is directly obtained by the combination of the modes computed from (29) with the corresponding eigenvectors of the cross correlation matrix defined in (26). Moreover, the total kinetic energy (TKE) of the data set is preserved, even if the energy of the modes is redistributed with respect to the previous variants presented.

\subsection{Mixed Fourier-Empirical decomposition}

The introduction of temporal and spatial normalization terms in the NE-POD procedure enables a corresponding Fourier-based decomposition of the modes, as in the classic POD (see e.g. [6, 23]). Denoting by $\tilde{U}=U W_{T}$ and by $\tilde{V}=V Z$, with $U, V, Z$ defined in the Eq. (29), let $\tilde{u}_{i}$ be the i-th column of $\tilde{U}$ (corresponding to the PIV data at the time instants $t_{i}$ ) and $\tilde{v}_{k}^{i}$ be the value contained in the i-th row and k-th column of $\tilde{V}$. Writing the relation (29) in the index notation, the k-th NE-POD mode (contained in the k-th column of the NE-POD mode matrix $\Phi$ ) can be written in the following way

$$
\phi_{k}=\sum_{i=1}^{N} \tilde{u}_{i} \tilde{v}_{k}^{i}=\sum_{i=1}^{N}\left(\sum_{n=1}^{N} \widehat{(\tilde{\tilde{u}})^{n}} e^{j \omega_{n} t_{i}}\right) \tilde{v}_{k}^{i}
$$

where $j$ is the imaginary number. The discrete values $\tilde{u}_{i}$ have been decomposed in time using the discrete Fourier transform with Fourier coefficients $\left(\widehat{\tilde{u}}^{n}\right.$ and angular frequency $\omega_{n}$. Exchanging the two summations, we obtain 


$$
\phi_{k}=\sum_{n=1}^{N} \widehat{(\tilde{u})^{n}}\left(\sum_{i=1}^{N} \tilde{v}_{k}^{i} e^{j \omega_{n} t_{i}}\right)
$$

Finally, since the second summation in (31) corresponds to the conjugate of Fourier coefficients of $\tilde{v}_{k}^{i}$, we have

$\phi_{k}=\sum_{n=1}^{N} \widehat{(\widetilde{\tilde{u}})^{n}}\left(\widehat{\left.\tilde{v}_{k}\right)^{*}}\right.$

Equation (32) shows that each NE-POD mode can be obtained as a combination of orthogonal functions that provides the sinusoidal content of each dynamics and the related temporal coefficients. Note that the energy of each mode is preserved if the whole spectrum is considered for the mode reconstruction. The procedure proposed here differs from the multi-scale POD approach of Mendez et al. [28]. In the multi-scale POD, the cross-correlation matrix defined in Eq. (3) is filtered a priori based on multi-resolution analysis. Then, POD modes are computed providing statistics of each frequency band considered. In the present method, the Fourier contributions to the modes are obtained combining the filtered snapshots and temporal coefficients obtained from the original not-filtered cross-correlation matrix. The capability of this technique to further highlight and isolate turbulent events not directly observed in the NE-POD modes will be further pointed out in the following sections.

\section{Application to numerical data}

The different POD variants presented in the previous sections were first applied to DNS data describing the organized vortex shedding behind a circular cylinder at a flow Reynolds number of 100 . The data set consists of 150 snapshots sampled at 10 times the temporal resolution of the simulation, describing 5 shedding periods. The spatial domain has been solved with $450 \times 200$ spatial points with the numerical scheme described in the work of Taira and Colonius [40]. The time-mean velocity and the rms distributions are reported in Fig. 1 with the aim of showing the spatial region interested by the cylinder wake, and the magnitude of velocity rms into this flow region. Further details on the boundary conditions and grid refinement can be found in the works of Kutz et al. [18]

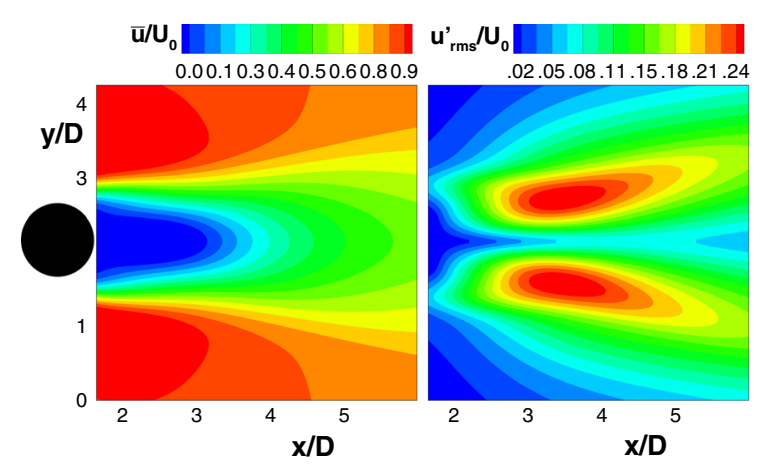

Fig. 1 (Left) time-mean normalized velocity $\bar{u} / U_{0}$ and (right) rms of velocity fluctuations $u_{r m s}^{\prime} / U_{0}$. Streamwise and cross-flow coordinates are scaled with the cylinder diameter $D$

and Brunton et al. [5], where the POD modes for this ensemble of data are also presented.

The different variants of POD discussed in the previous section are applied to the present data set to highlight the role of the different weights and scalar product applied to a well known, established, noise free case, where only the large scale rolls in the cylinder wake are present. The distribution of the normalized energy of the original field reconstructed with the first 10 modes for the different cases are reported in Fig. 2. Since the classic POD, TI-POD, SIPOD and TSI-POD are all characterized by a symmetric cross-correlation matrix, i.e. their modes are orthogonal with respect to the Euclidean norm, the sum of the eigenvalues directly provides the percentage of kinetic energy associated with the related modes. However, based on the definition of the POD

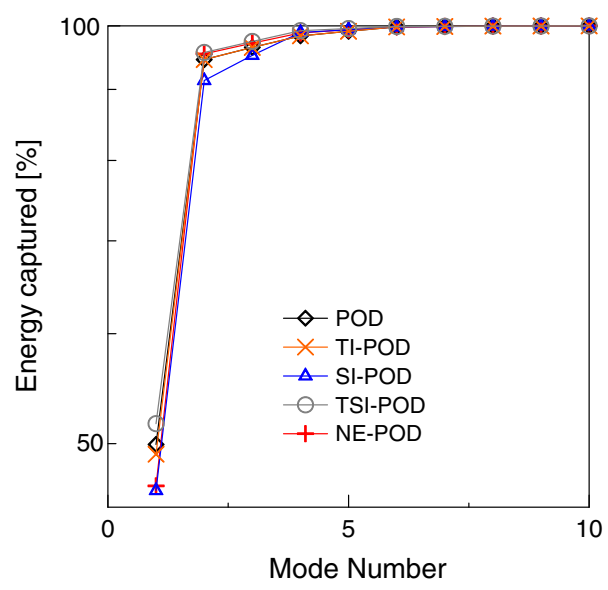

Fig. 2 Energy content of reconstructed fields obtained with the different procedures 
kernel of the different variants, the summation of the eigenvalues provides the real kinetic energy of the flow only for the classic POD, since in the other cases the sum of the eigenvalues corresponds to the kinetic energy of the normalized field $\tilde{U}$. Additionally, for the NE-POD, due to the not-symmetric correlation matrix, the energy content of the fields reconstructed with a specific number of NE-POD modes differs from the sum of the energy captured by the same modes (i.e. the sum of the corresponding eigenvalues). Figure 2 shows that for this organized flow pattern, the second order truncation accounts for an overall energy in the surround of $95 \%$ for all the procedure except for the SI-POD, that recovers the others after the fourth mode.

The spatial distributions of the first mode obtained with the 5 different procedures are shown in Fig. 3. These modes are obtained by projection of the weighted snapshot matrix $\tilde{U}$, which is defined depending on the different variants. The first mode provides the statistical representation of the largest scale rolls dominating the instability of the cylinder wake (see for example [18, 31]). The classic POD and the TI-POD exhibit practically identical results in this particular case concerning a strong statistically stationary flow (i.e. the diagonal of $W_{T}$ is almost constant and the normalization step provided by the TI-POD does not redistribute the energy of the different snapshots). Conversely, the SI-POD and the TSI-POD provide substantially different modal representations. The cylinder vortices appear significantly enlarged with respect to the POD modes, extending also outside of the wake region. This is due to the spatial normalization introduced in these two procedures. The spatial weighting matrix introduced in TSI and SI-POD methods may therefore lead to a wrong estimation of the main scales embedded within the flow, which represents a significant shortcoming when they would be used to construct reduced order models. The increased optimality of the present methods with respect to the classic POD are ascribed here to the fictitious enlargement of the finer scales, acting redistributing the overall energy of fluctuations toward the lower order modes (describing large scale coherent structures). It shoukd be noted also that the TSI and the SI-POD methods show the same results due to the low variance of the diagonal entries of $W_{T}$ appearing in the TSI-POD, where the spatial normalization clearly dominates. On the other hand, the last plot of Fig. 3 shows that the inclusion of a different definition of the scalar product in the NE-POD procedure does not introduce significant modification of the main scales embedded within the flow, showing the same results provided by the classic POD and TI-POD. In the following experimental cases the NE-POD procedures will be shown to provide different modal representations of the data ensembles, since information into the snapshots includes a multitude of turbulent events.

\section{Application to experimental data}

In order to better highlight the peculiarities of the different methods, experimental data describing laminar to turbulent transition for attached and separated flows developing on a flat plate under high free-stream turbulence intensity (FSTI) level are considered in this section. The chosen conditions provide evidence of the capability of the different procedures in case of strong not uniform flows (in space), and with a more sparser occurrence of turbulent events in time with respect to the organized vortex shedding previously discussed. Basically, also for the following applications the TIPOD shows the same results of the classic POD. This is because the normalization matrix $W_{T}$ in Eq. (9) has diagonal entry elements that are almost constant (the variance normalized by the mean value is 0.1 ). A significant energy redistribution between the rows of

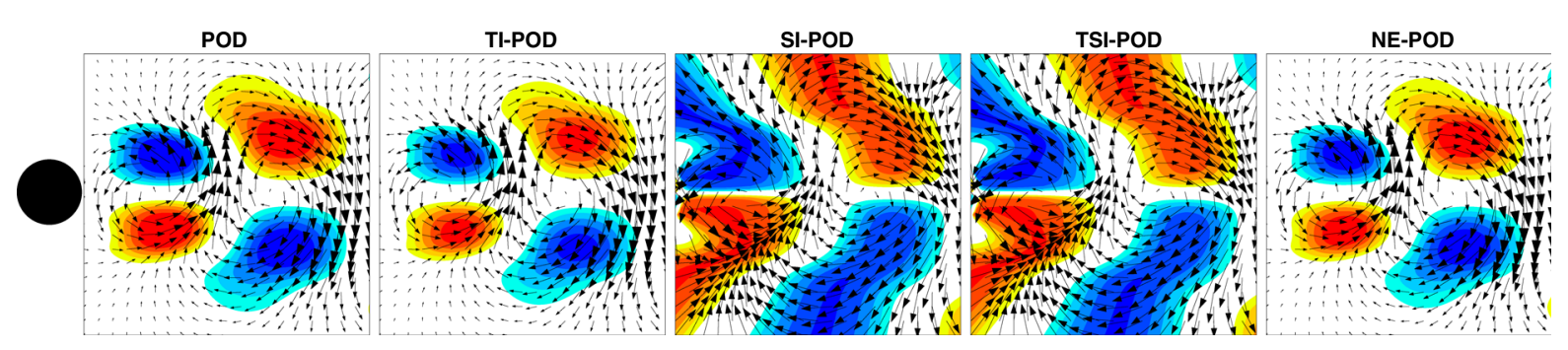

Fig. 3 Vector fields of the first mode obtained by the different procedures 
the snapshots matrix is instead done by the spatial weighting matrix $W_{S}$, due to the high spatial nonhomogeneity of the flows at hand (the rms of the diagonal entry elements of $W_{S}$ is significantly greater than that of $W_{T}$ ). This means that also in these cases, the effects of the weighting matrix $W_{S}$ dominate, thus the TSI-POD and the SI-POD provide the same modal representation of the flow. Then, only the modes of the POD, SI-POD and NE-POD are presented in this work. Application of the TI-POD would be of great interest in the case of phase dependent data such as those of Lengani et al. [21], providing different modal representations.

The experimental data were acquired in a double contoured test section producing a strong adverse pressure gradient (APG) to the flow, typical of ultra high lift blade profile (Fig. 4, see [38] for further details). The plate is $200 \mathrm{~mm}$ long and $300 \mathrm{~mm}$ wide to ensure two dimensional time-mean flow at midpsan of the plate. An elliptic leading edge has been used to avoid flow separation in this part of the plate. Data have been acquired in the meridional plane by means of a DANTEC time resolved PIV system (with a sampling of frequency $3168 \mathrm{~Hz}$ ) for about 1s, collecting sequences of 3100 evenly time spaced instantaneous PIV snapshots. Such amount of data provides a good statistical convergence for the most energetic POD modes. The instrumentation is constituted by a dual-cavity Nd:YLF pulsed laser Litron LDY 300 (maximum energy per pulse $30 \mathrm{~mJ}$ at $1000 \mathrm{~Hz}$ repetition rate, $527 \mathrm{~nm}$ wavelength). The adaptive cross-correlation algorithm has been used with a finer interrogation area of $16 \times 16$ pixels and $50 \%$ overlap. This gives a uniform spatial measuring grid with a distance between adjacent vectors of $0.43 \mathrm{~mm}$. This measuring grid allows solving with at least 10 measuring points the large scale structures originating in both the conditions tested, thus ensuring a good convergence of the most energetic POD modes according to Tinney et al. [41]. A peak validation

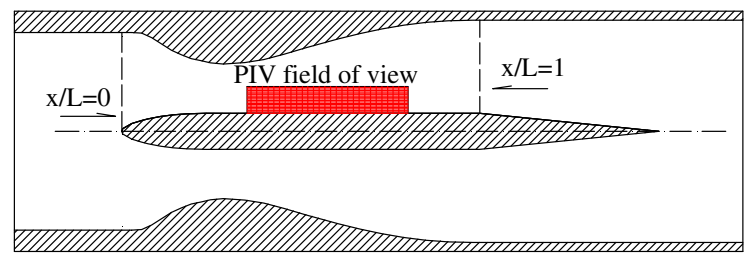

Fig. 4 Test section and PIV field of view has been used to discriminate between valid and invalid vectors.

\subsection{Bypass transition}

The first experimental condition examined concerns an attached flow undergoing laminar to turbulent transition beneath high FSTI level (2.87\%). In this context, boundary layer streaks are known to drive the transition process. The Reynolds number based on the inlet velocity and the plate length $L$ is 100,000 . At this Reynolds number the boundary layer did not separate despite the strong APG imposed to the flow, as further documented in the previous authors' work Simoni et al. [38]. The normalized streamwise velocity $\bar{u} / U_{0}$ and velocity $\mathrm{rms} u_{r m s}^{\prime} / U_{0}$ distributions are reported in Fig. 5, with $U_{0}$ the free-stream velocity at the measuring domain inlet. The boundary layer thickness sensibly grows in the downstream direction as a consequence of the strong APG. Moreover, the increase of $u_{r m s}^{\prime} / U_{0}$ (right plot of Fig. 5) and its further reduction suggest that the flow undergoes laminar to turbulent transition. The boundary layer is in a fully turbulent condition downstream of $x / L \cong 0.58$, as shown by the intermittency function distribution reported in Simoni et al. [36].

The energy content of reconstructed fields obtained from an increasing number of modes for the classic, the SI-POD and the NE-POD procedures is presented in Fig. 6. In this case both SI-POD and NE-POD methods lose optimality with respect to the classic POD, with the NE-POD energy distribution relaying in between the other two curves. After the first 10 modes the curves do not differ by more than $10 \%$ and by no more than $2 \%$ after 100 modes. Note that in simple or at least slightly complex flows, like in the wake of the circular cylinder of the previous example, a small number of modes well reproduce the flow field dynamics. However, for strongly inhomogeneous flows (as that of the present data-set) a higher number of modes are typically required to capture a significant amount of energy of the flow, as also discussed in Glauser and George [13].

The most energetic modes provided by the POD, SI-POD and NE-POD are described in Figs. 7, 8 and 9, respectively. These figures show the odd modes of the first 12, carrying an overall energy between $43 \%$ (SIPOD) and 50\% (POD) for the different procedures. 

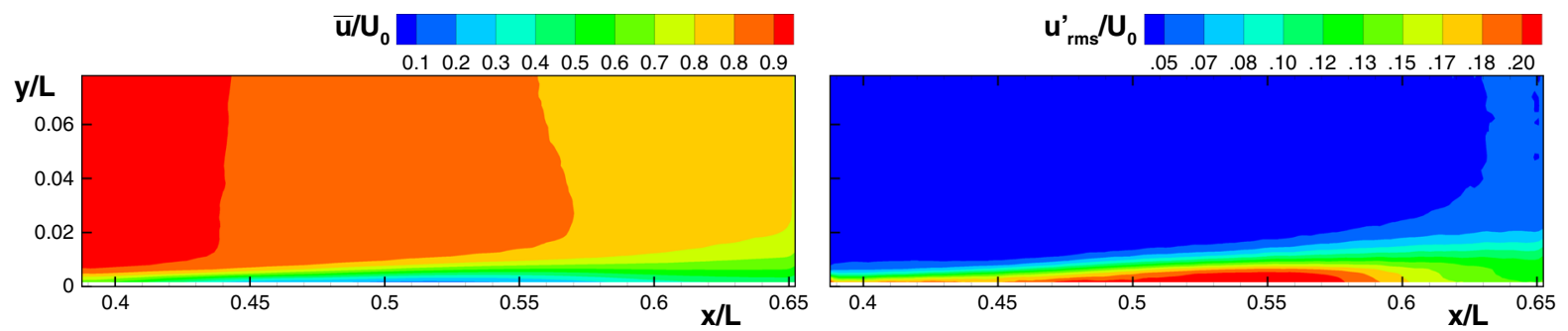

Fig. 5 (Left) time-mean normalized streamwise velocity $\bar{u} / U_{0}$ and (right) rms of velocity fluctuations $u_{r m s}^{\prime} / U_{0}$. Streamwise and the normal to the wall coordinates are scaled by the plate length $L$

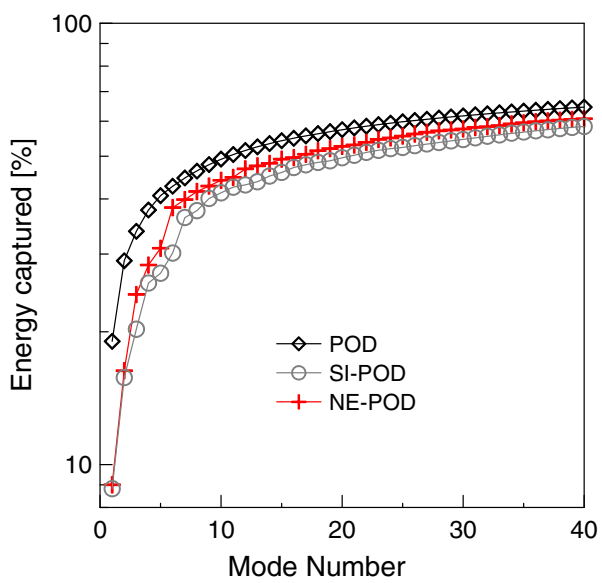

Fig. 6 Energy content of reconstructed fields obtained from an increasing number of POD, SI-POD and NE-POD modes for bypass transition case

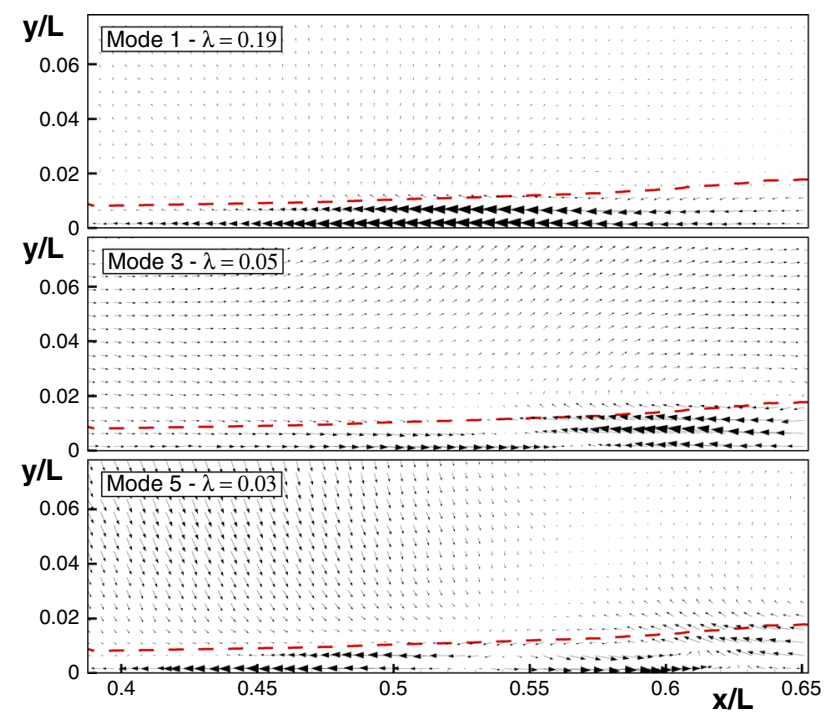

The POD modes shown in Fig. 7 clearly show large streamwise fluctuations in the boundary layer typical of by-pass transition. The first classic POD mode shows streamwise velocity fluctuations within the boundary layer region where the highest values of $u_{r m s}^{\prime} / U_{0}$ are observed in Fig. 5. This is a typical statistical representation of elongated boundary layer streaks. Conversely, the third mode shows vectors pointing upstream close to the transition end position $(x / L=0.6)$, and a perturbation velocity vortex is recognizable at the edge of the boundary layer. In these modes, the classic POD method isolates the most energetic boundary layer fluctuations from the freestream turbulence, as a consequence of their significantly different energy content. Events resembling free-stream vortices are only observable in modes 7

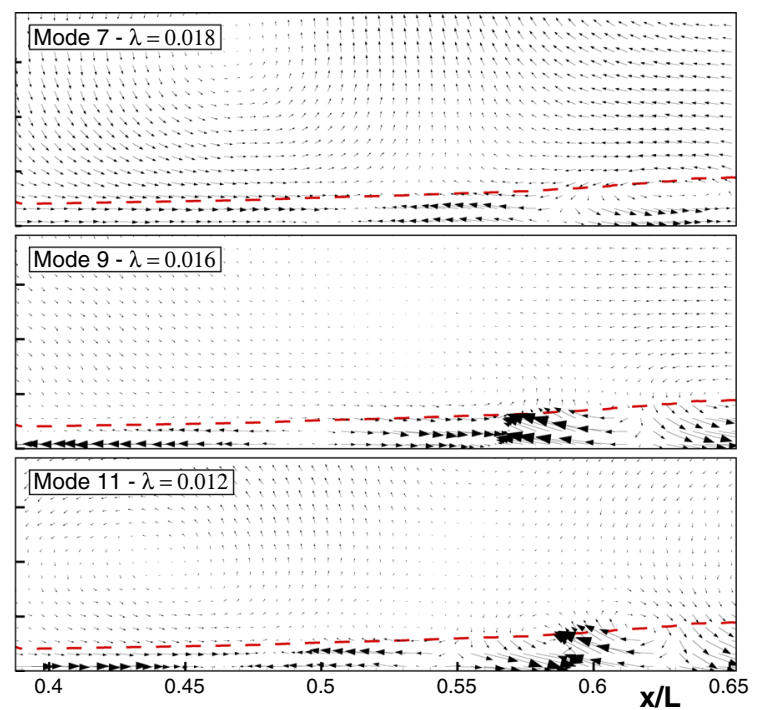

Fig. 7 Vector fields of the first odd 12 classic POD modes. Mode number and corresponding eigenvalue is indicated in the upper-left corner of each plot. Mean boundary layer thickness is indicated by red line 

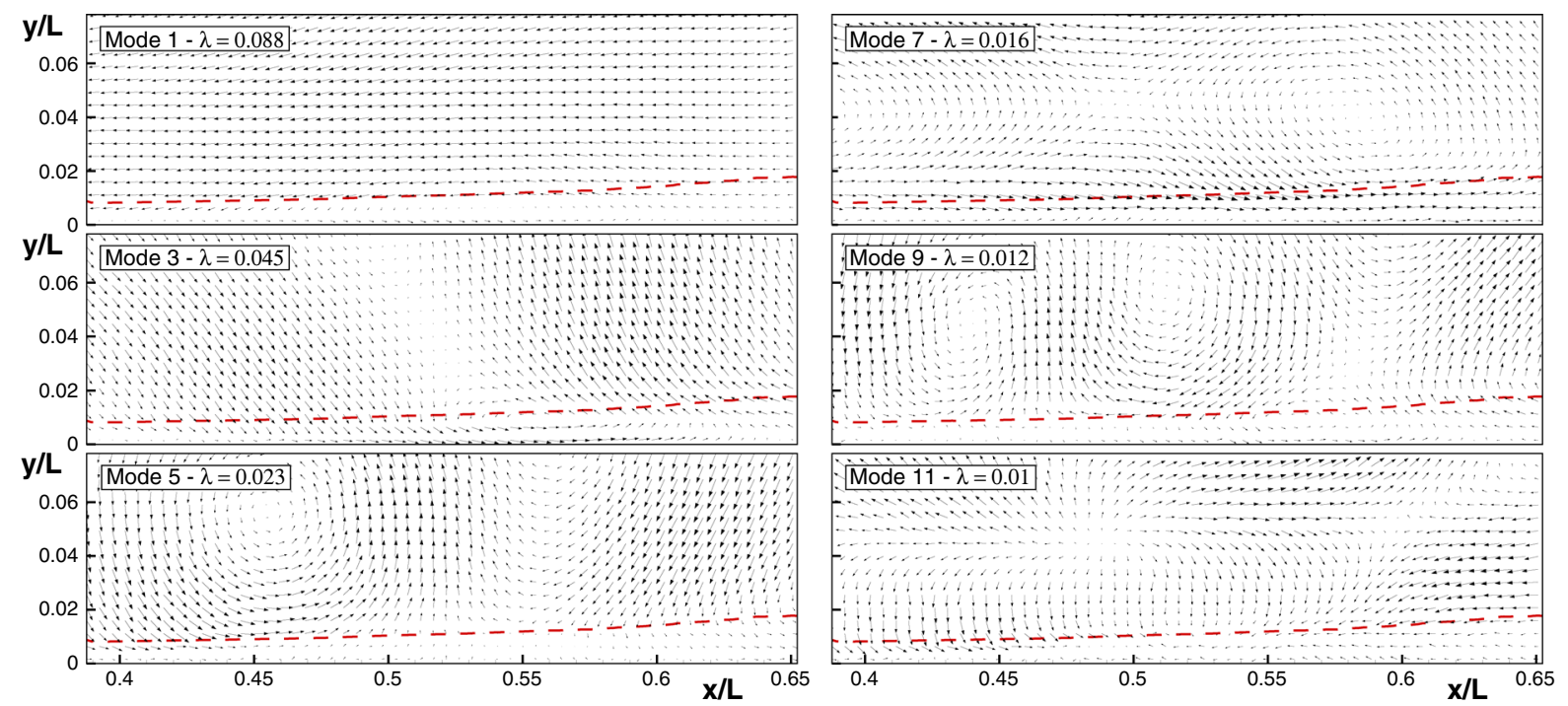

Fig. 8 Vector fields of the first odd 12 SI-POD modes. Mode number and corresponding eigenvalue is indicated in the upper-left corner of each plot. Mean boundary layer thickness is indicated by red line
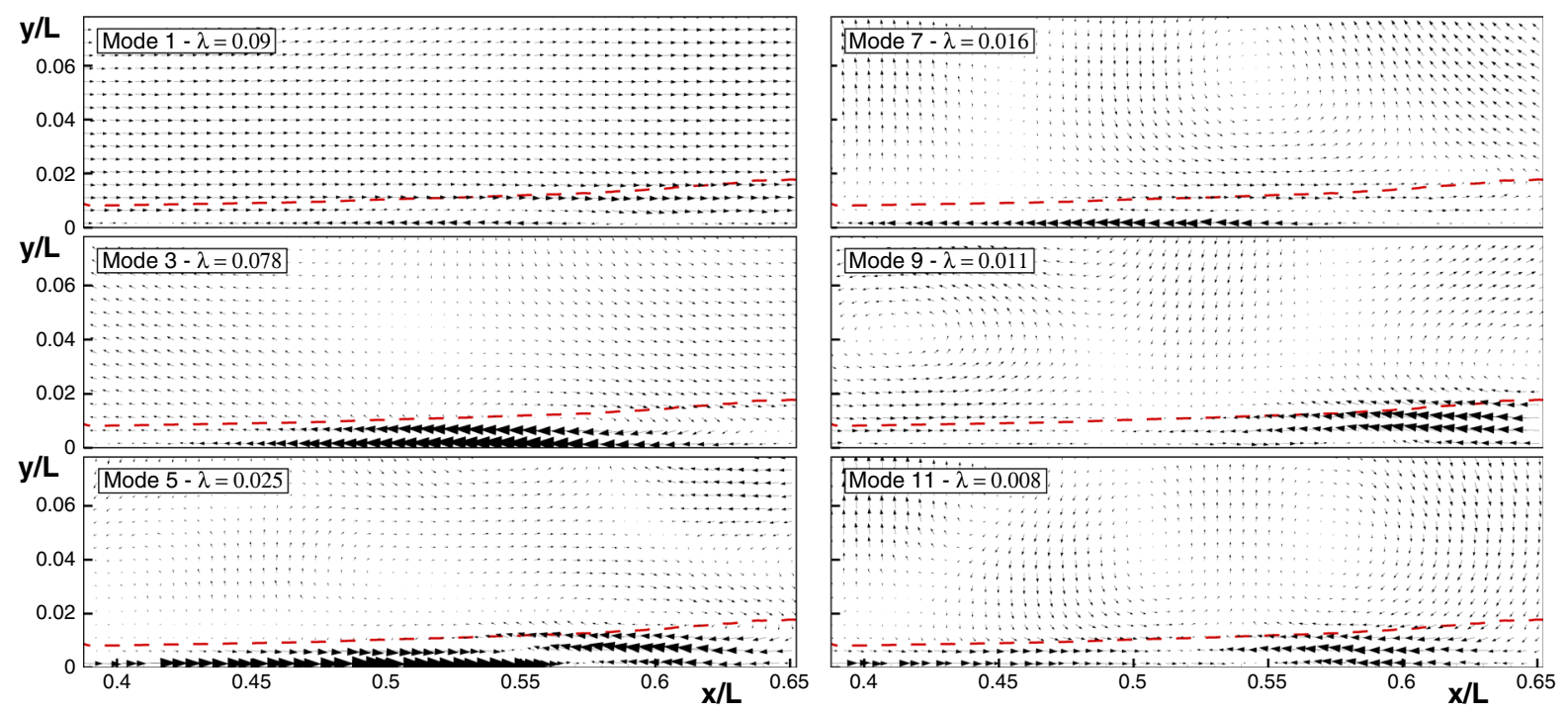

Fig. 9 Vector fields of the first odd 12 NE-POD modes. Mode number and corresponding eigenvalue is indicated in the upper-left corner of each plot. Mean boundary layer thickness is indicated by red line

and 11, where boundary layer fluctuating activities are less pronounced.

The spatial normalization introduced into the SIPOD makes free-stream oscillations more evident than in the snapshot POD modes. Indeed, all the modes of Fig. 8 better highlight turbulent events in the freestream, resembling large scale vortices that are known to play a crucial role in the breakup of streaky structures, leading to the by-pass transition of the boundary layer (e.g., [17]). Modes 5 and 9 clearly resemble a train of vortices at the edge of the time-mean boundary layer. However, the SI-POD procedure completely loses information about boundary layer events (due to the occurrence of streaky structures), since they are sparse events occurring randomly in time, hidden by the introduction of the spatial normalization in Eq. (18).

The possibility to take advantage of both spatial normalization, without precluding the possibility to 
highlight sparse events embedded in the data ensemble, is the main characteristic of the NE-POD method. Large scale vortices at the edge of the boundary layer are observable in the NE-POD modes 7, 9 and 11, but in this case boundary layer fluctuations are also represented by the modes. Particularly, the 3-rd and 5-th NE-POD modes show high fluctuations in the boundary layer resembling boundary layer streaks (similar to the first POD mode of Fig. 7), with also free-stream oscillations. However, in these modes the free-stream fluctuations do not assume the shape of large scale vortices, even though the following ones suggest their presence.

In order to further exploit the capability of the NEPOD method to capture free-stream and boundary layer related events, modes 3 and 5 of the NE-POD were further decomposed as a linear combination of pure sinusoidal contributions. This provides a further split of the different fluctuations based on their frequency content. The FFTs of the 3-rd and the 5-th NE-POD temporal coefficients have been computed first to highlight the frequency content of the turbulent events captured by the corresponding modes (see Fig. 10). A low frequency peak with rapid energy decrease characterizes the spectrum of the third mode (rhombus point symbols), while a broader band spectrum characterizes the fifth mode (circle point symbols). Thus, mode 3 mainly describes low-frequency events while modes 5 is related to multiple frequencies. Two bands have been therefore considered for the decomposition of the modes by Eq. (32). More precisely, the frequency capturing similar energy in both spectra (i.e. where the spectra intersect) was chosen to distinguish between low and high

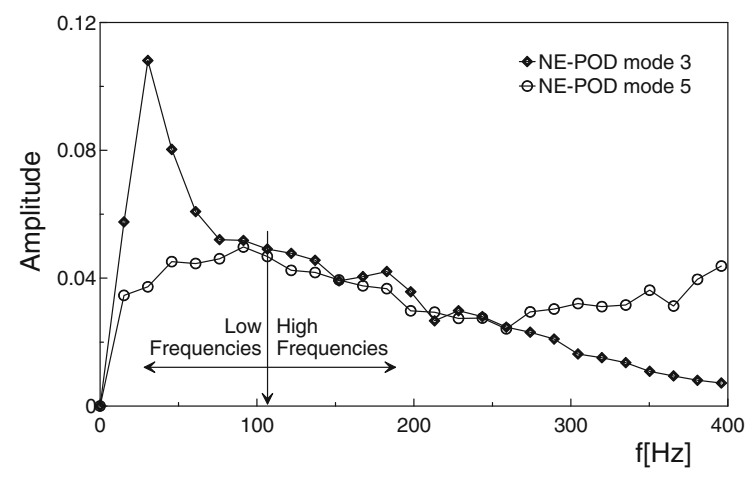

Fig. 10 FFTs of eigenvectors corresponding to NE-POD modes 3 (rhombus point symbols) and 5 (circle point symbols) frequency contributions, as shown in Fig. 10. The low frequency activity mostly characterizing the third NEPOD mode is therefore isolated from the higher frequencies animating the fifth mode. It should be noted that the frequency bands used for the mode decomposition are strictly related to the specific flow configuration at hand.

The low frequency contributions to both NE-POD modes are reported in Fig. 11 (top plots). They are related to the occurrence of boundary layer fluctuations for both the modes presented. On the contrary, in the present case the higher frequencies contributions (bottom plots of Fig. 11), mostly describe events related to free-stream vortices which are located above the boundary layer edge, showing similarities with the shear sheltering mechanism well described by Jacob and Durbin [17] and successively by Zaki and Saha [46]. To the authors' knowledge no evidence of this phenomenon has been yet obtained from experimental data by means of the existing modal decomposition techniques. The weights introduced in the new procedure act enhancing sparse events occurring in time (i.e. boundary layer streaks) and turbulent events embedded in low mean kinetic energy, like free-stream vortices that are typically not captured by other procedures. In the bottom-right plot of Fig. 11, $Q_{2}$ events (i.e. $u^{\prime}<0$ and $v^{\prime}>0$, according to [30]) around $x / L=0.6$ are also more evident than in the original NE-POD modes, coexisting with free-stream vortices animated by the same frequencies. On the other hand, bursting events are instead not observable in the bottom-left plot of this figure, where free-stream structures are evidently characterized by larger length scale.

\subsection{Separated flow transition}

The second experimental data set concerns a pressure induced laminar separation bubble. This transitional environment shares a great not uniformity with the bypass case, jointly with an organized vortex shedding phenomenon similar to the DNS data set. Measurements have been carried out in the same test section (Fig. 4) with the same inlet FSTI $(2.87 \%)$ of the previous data set. The Reynolds number based on the inlet velocity and the plate length $L$ is instead reduced to 40,000 . In this context a laminar separation bubble occurs as a consequence of the strong APG imposed to the flow. In such flow configuration, the amplification 

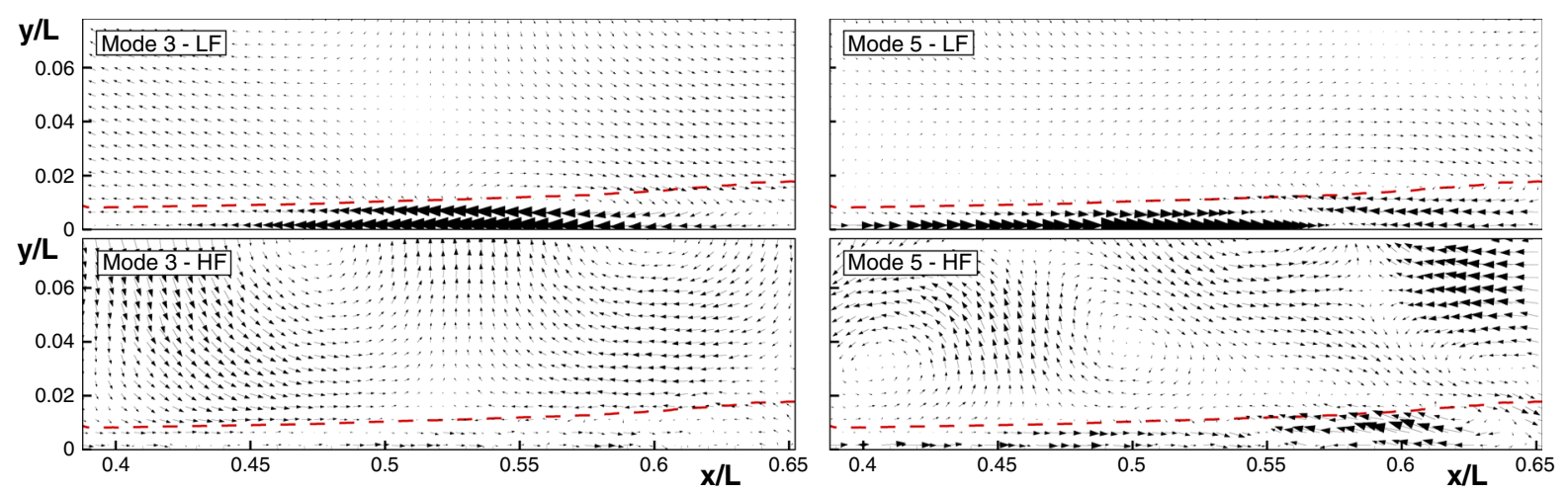

Fig. 11 Low frequency (LF) and high frequency (HF) contributions to NE-POD modes 3 and 5. Mode number is indicated in the upperleft corner of each plot. Mean boundary layer thickness is indicated by red line. (Color figure online)

of velocity fluctuations due to the Kelvin-Helmholtz instability process drives the formation of large scale roll-up vortices forcing transition, thus reattachment, as described in the works of Diwan and Ramesh [9], Marxen and Henningson [25] and Michelis et al. [29].

The contour plots of the normalized streamwise velocity $\bar{u} / U_{0}$ and the corresponding $\mathrm{rms} u_{r m s}^{\prime} / U_{0}$ are reported in Fig. 12. The flow region with negative streamwise velocity confirms the occurrence of a separation bubble (blue region in the plot). The separation and the maximum displacement positions are $x / L=0.39$ and $x / L=0.52$, respectively. These positions were determined by looking at the mean flow structure and the boundary layer integral parameters, as described by Simoni et al. [38]. Maximum values of the $u_{r m s}^{\prime} / U_{0}$ are localized inside the separated shear layer, along the velocity profile inflection line, and the maximum turbulence intensity was found in the flow region downstream of the bubble maximum displacement position, as also shown in the work of Yang and
Voke [43]. The high velocity fluctuations observable in the reattachment region are due to the occurrence of the large scale structures shed as a consequence of the shear layer roll-up.

As for the previous cases, the energy content of reconstructed fields obtained from an increasing number of modes for the classic POD, the SI-PDO and the NE-POD procedures is presented first in Fig. 13. Also in this case both the SI-POD and the NEPOD lose optimality with respect to the classic method, with the NE-POD distribution being closer to the SI-POD one. The difference between the curves is $6 \%$ after 10 modes and they differ again by no more than $2 \%$ after 100 modes.

The odd modes of the first 12 are reported in Figs. 14, 15 and 16 for the classic POD, the SI-POD and the NE-POD, respectively. Only the odd modes have been chosen for the sake of brevity. The first POD mode of Fig. 14 exhibits streamwise fluctuations in the whole bubble, while the third one shows negative

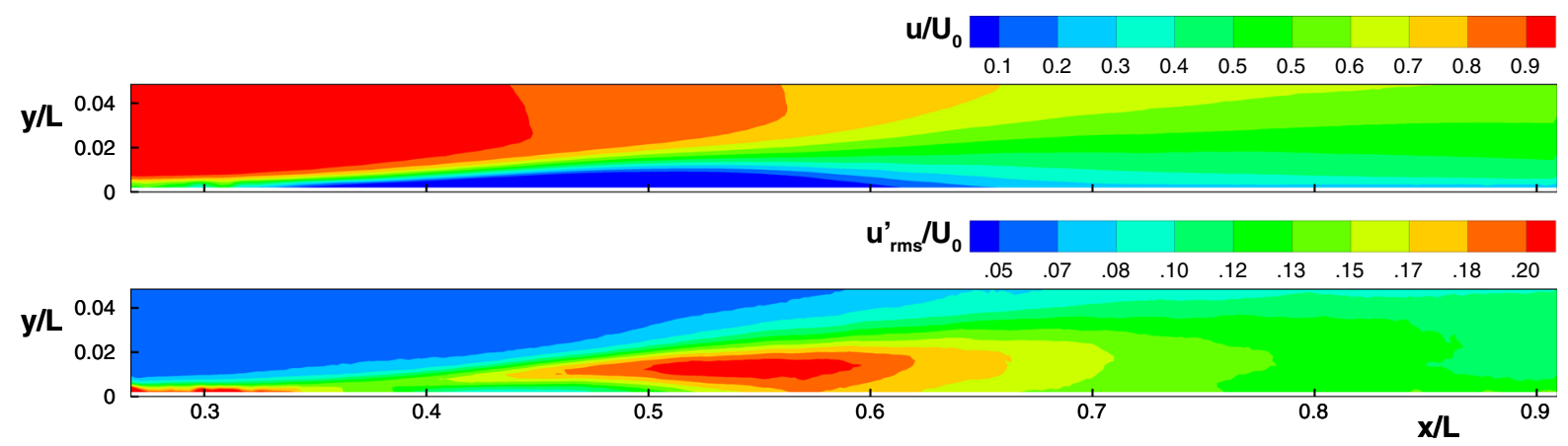

Fig. 12 (Top) time-mean normalized streamwise velocity $\bar{u} / U_{0}$ and (bottom) rms of velocity fluctuations $u_{r m s}^{\prime} / U_{0}$. Streamwise and the normal to the wall coordinates are scaled by the plate length $L$ 


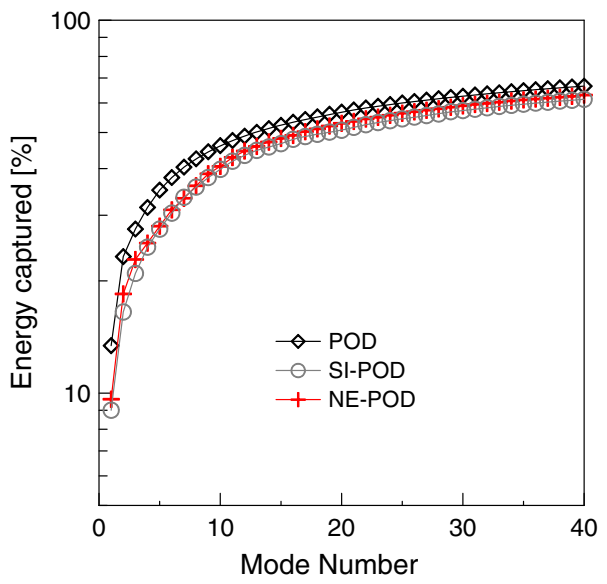

Fig. 13 Energy content of reconstructed fields obtained from an increasing number of POD, SI-POD and NE-POD modes for separation induced transition

vectors in the fore part of the separated shear layer with large scale fluctuations occurring behind the maximum displacement position $(x / L \approx 0.67$, $y / L \approx 0.04)$. These modes may be linked to the motions of the whole bubble due to the feed-back loop mechanism and mean flow deformation (see [26]), or to the presence of streaky structures in the separated shear layer, due to the quite high FSTI level. Mode 5 and successive ones represent a sequence of counterrotating vortices generating downstream of $x / L=0.58$, while the POD vectors are significantly smaller upstream of this position. These modes are representative of the shedding process dominating the rear part of the bubble, as well described in the works of Diwan and Ramesh [9] and Marxen and Henningson [25].

The modes obtained by the SI-POD method are reported in Fig. 15. With respect to the classic POD, the spatial normalization introduced in Eq. (18) strongly emphasizes free-stream oscillations above the separated shear layer, as in both previous cases. Turbulent events into the separated shear layer (such as those observed in the classic POD mode 3) are not shown by the modes. As for the by-pass case, sparse events in time are not captured by the SI-POD modes, which mainly depict free-stream fluctuations and K-H rolls. Vortices in the reattaching part of the boundary layer are observable in modes 7, 9 and 11, while oscillations in the free-stream region assume the shape of large scale vortices only in mode 7.

Again, as also observed in the case of the by-pass transition, the weighted scalar product introduced into the definition of the NE-POD method allows capturing turbulent events resembling streaky structures in the

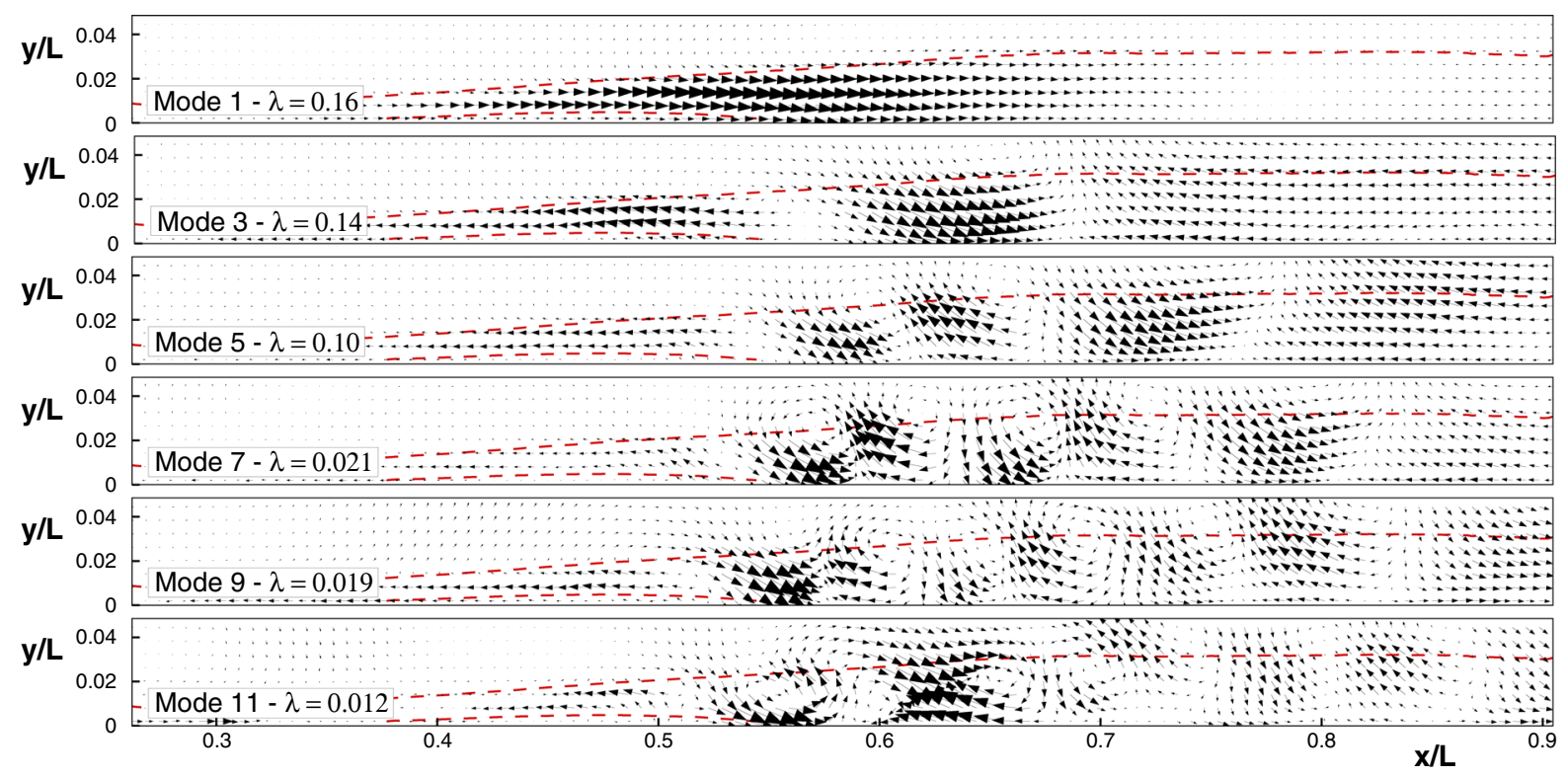

Fig. 14 Vector fields of the first odd 12 classic POD modes in the separated case. Mode number and corresponding eigenvalue is indicated in the bottom-left corner of each plot. Mean flow structure is highlighted by iso-contour lines of the mean velocity (red lines). (Color figure online) 


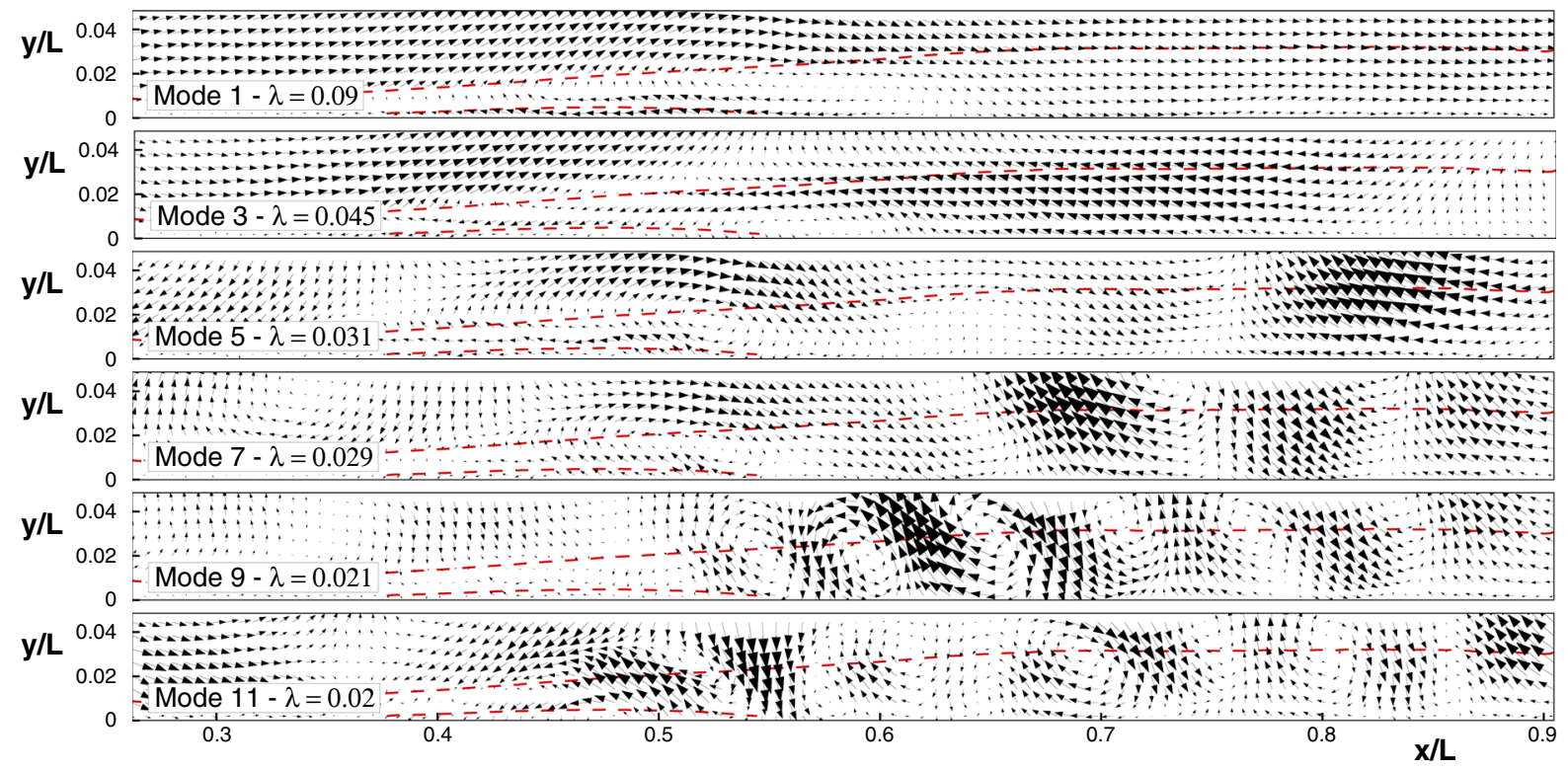

Fig. 15 Vector fields of the first odd 12 SI-POD modes in the separated case. Mode number and corresponding eigenvalue is indicated in the bottom-left corner of each plot. Mean flow structure is highlighted by iso-contour lines of the mean velocity (red lines). (Color figure online)

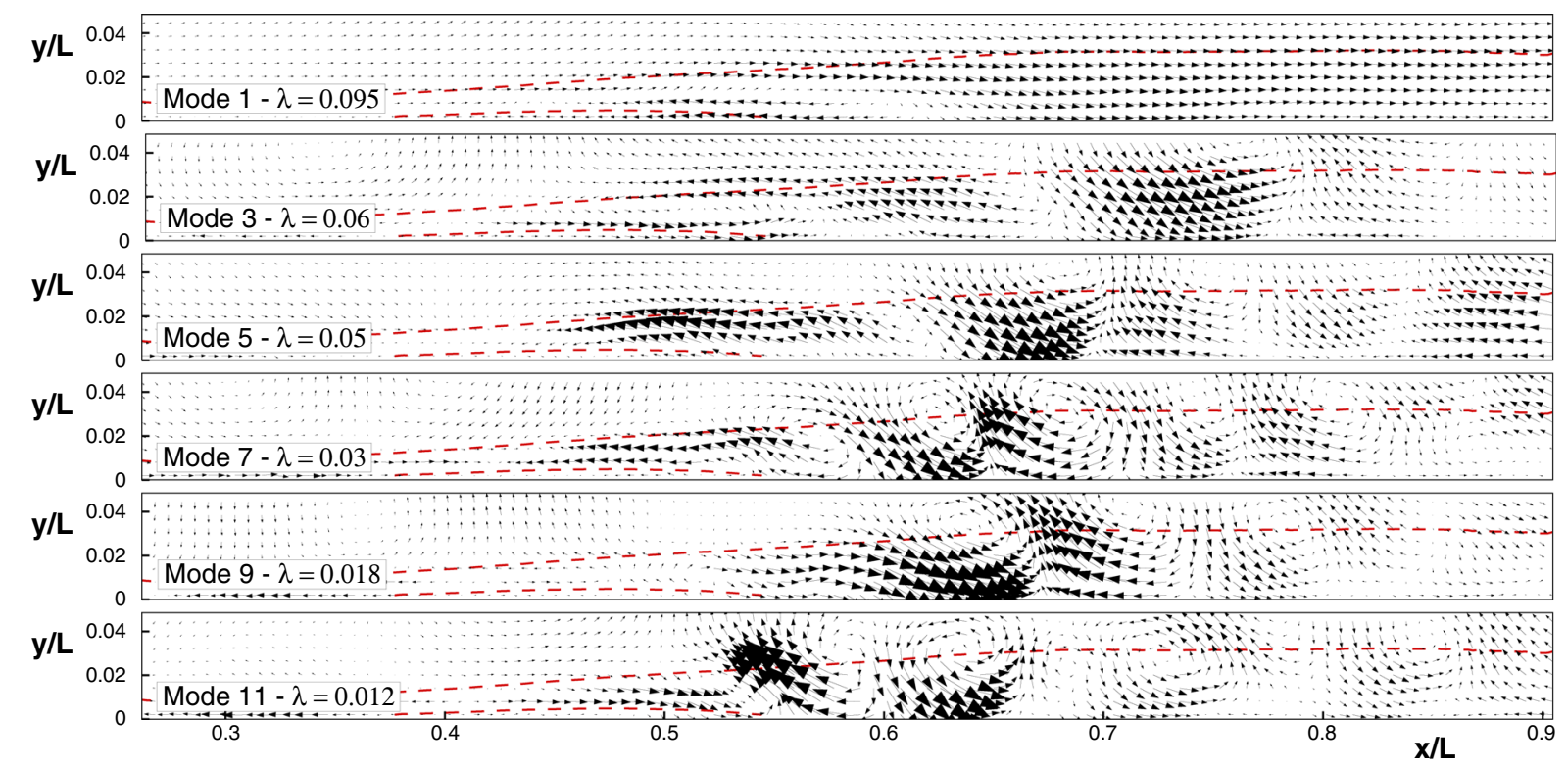

Fig. 16 Vector fields of the first odd 12 NE-POD modes in case of separated boundary layer. Mode number and corresponding eigenvalue is indicated in the bottom-left corner of each plot. Mean flow structure is highlighted by iso-contour lines of the mean velocity (red lines). (Color figure online)

fore part of the bubble as well as free-stream vortices. Indeed, modes 5 and 7 of Fig. 16 clearly exhibit streamwise oscillations in the fore part of the bubble between $x / L=0.46$ and $x / L=0.58$, while also emphasizing flow oscillations in the free-stream region upstream of $x / L=0.45$ (see mode 7). Oscillation events resembling free-stream vortices are also visible in modes 3,9 and 11. The less energetic oscillation events occurring upstream of the bubble maximum displacement position are also more 
highlighted with respect to the classic POD. Also in this case, the Fourier-based decomposition procedure described in Sect. 2.6 was applied to further isolate the turbulent events captured by the NE-POD modes. To this end, NE-POD modes 5 and 7 are decomposed since they show the coexistence of streamwise fluctuations in the separated shear layer, $\mathrm{K}-\mathrm{H}$ rolls and also free-stream fluctuations above the boundary layer edge.

The FFT of the 5-th and 7-th eigenvectors are reported in Fig. 17. Low frequency fluctuations mostly contribute to mode 5 (rhombus point symbols), while mode 7 is animated by a temporal coefficient with deterministic higher frequencies, with the peak linked to the characteristic shedding frequency of the separated shear layer, as described in details in the previous authors' work Simoni et al. [38]. Note that the 5-th eigenvector still has a significant amplitude at this frequency. The frequency capturing similar energy in these two spectra has been chosen also in this case to distinguish between low and high frequency contributions to the modes. Low frequency contributions allow isolating velocity fluctuations in the fore part of the bubble (Fig. 18). Particularly, vectors pointing upstream are clearly observable in the low frequency contributions to the modes. This suggests that streaky structures may occur in the separated boundary layer due to the high FSTI level, according to what was shown in the work of Istvan and Yarusevych [15] for similar flow configuration. Otherwise, the counter rotating vortices shown in the original modes downstream of $x / L=0.6$ are not observable in these plots. Evidence of the shedding process are instead recognizable in the high frequency contributions to the

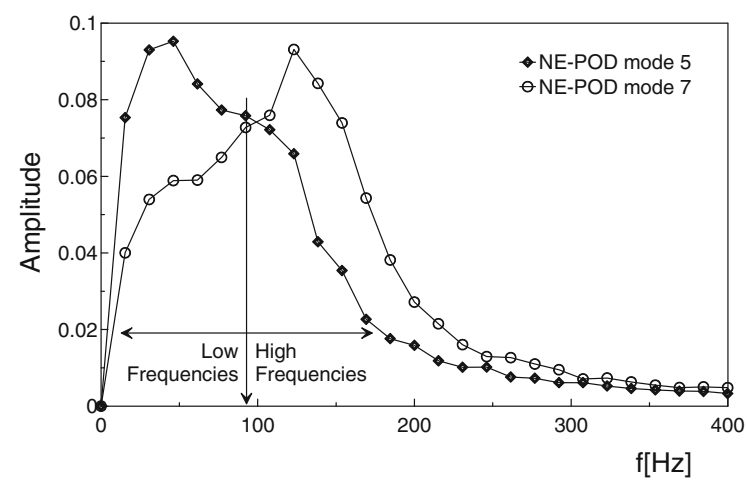

Fig. 17 FFTs of eigenvectors corresponding to NE-POD modes 5 (rhombus point symbols) and 7 (circle point symbols) modes. In both the second and fourth plots the turbulent events appear more organized than in the corresponding overall modes (compare Fig. 18 to 16). Additionally, high frequency contributions also highlight free-stream fluctuations above the separated shear layer (bottom plot of Fig. 18), thus sharing similarities with results obtained for the attached flow configuration in the foregoing example.

\section{POD and NE-POD filtered velocity fields}

In this section, velocity fields obtained as combination of a sub-set of classic POD and NE-POD modes are presented. The aim is to discuss the different flow features highlighted by these two low-order representations of the original data sets. It is pointed out here that the full rank reconstruction provides the original flow field for both the decompositions, since NE-POD was shown to preserve the original energy of the flow. Otherwise, considering only a subset of modes provides evidence of the redistribution of velocity fluctuations among the NE-POD spectrum with respect to the classic POD. Figures 19, 20 depict sequences of perturbation velocity maps for the bypass and separation induced transition, respectively. Velocity fields are reconstructed using 10 modes, for which the cumulative energy distributions reported in Figs. 6 and 13 differ for no more than $10 \%$.

Figure 19 shows 4 snapshots of NE-POD (left column) and POD (right column) filtered fields for the by-pass transition case. Note that the same sequence of snapshots is presented in such a way to directly highlight the different features captured by the low order representations of the present data set. Both the NE-POD and the POD filtered fields clearly highlight the occurrence of velocity fluctuations into the boundary layer (delimited with red line in the plots). For both the decompositions adopted, a low-speed streak is observed in the top snapshot around $x / L=0.52$, which is shown to break up in the successive images. The highest differences between NE-POD and the POD filtered fields are observed where streak breakup occurs $(x / L>0.52)$. Particularly, in the POD filtered snapshots (right column), $Q_{2}$ events are more highlighted than in the corresponding NE-POD filtered field. In the classic POD, breakup events, which are characterized by elevate energy of fluctuations, overshadow those linked to the formation 


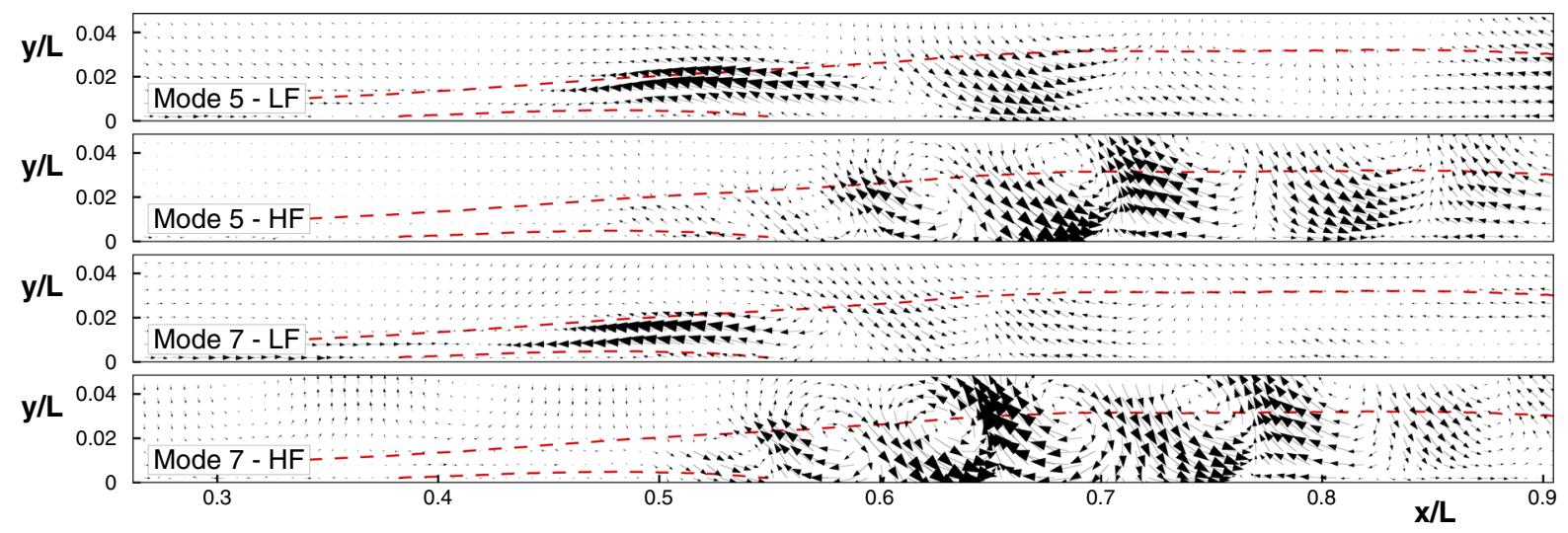

Fig. 18 Low frequency (LF) and high frequency (HF) contributions to NE-POD modes 5 and 7. Mode number is indicated in the bottom-left corner of each plot. Mean flow structure is highlighted by iso-contour lines of the mean velocity (red dashed line). (Color figure online)
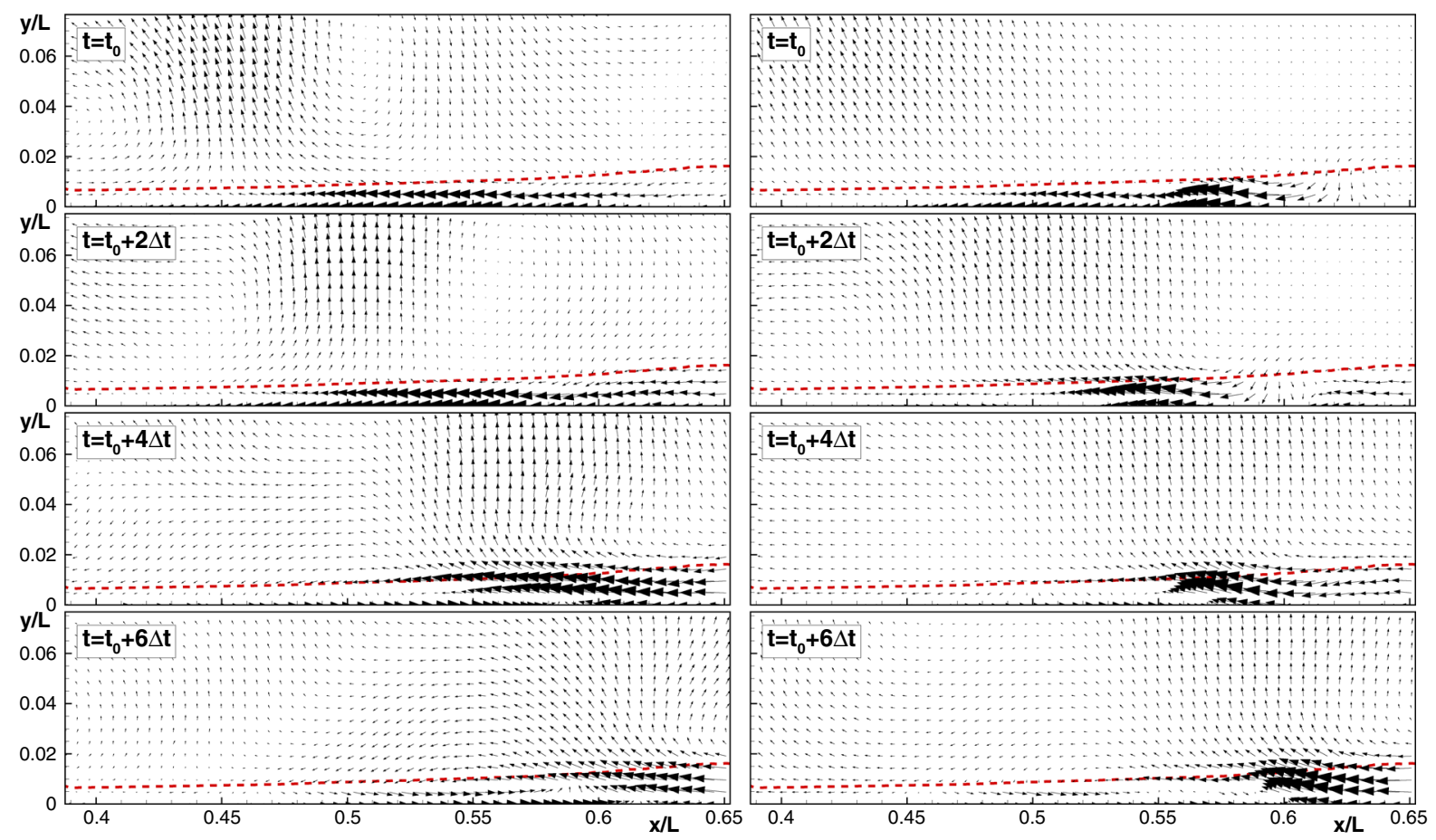

Fig. 19 Sequence of perturbation velocity maps for by-pass transition case reconstructed using 10 (left) NE-POD and (right) classic POD modes. Time-mean boundary layer thickness is highlighted by means of contour line of $u / U_{\infty}=0.99$ (red line). (Color figure online)

and propagation of streamwise oriented streaks, which are instead highlighted using the NE-POD modes for the reconstruction of the instantaneous velocity field. Additionally, free-stream fluctuations are better captured in the instantaneous realizations reported on the left column of Fig. 19. This is due to spatial weight matrix introduced in the NE-POD procedure. As a consequence, using NE-POD modes instead of the classic POD ones for the reconstruction of low order models of the flow field at hand allows for a better characterization of coexisting free-stream and streaks 

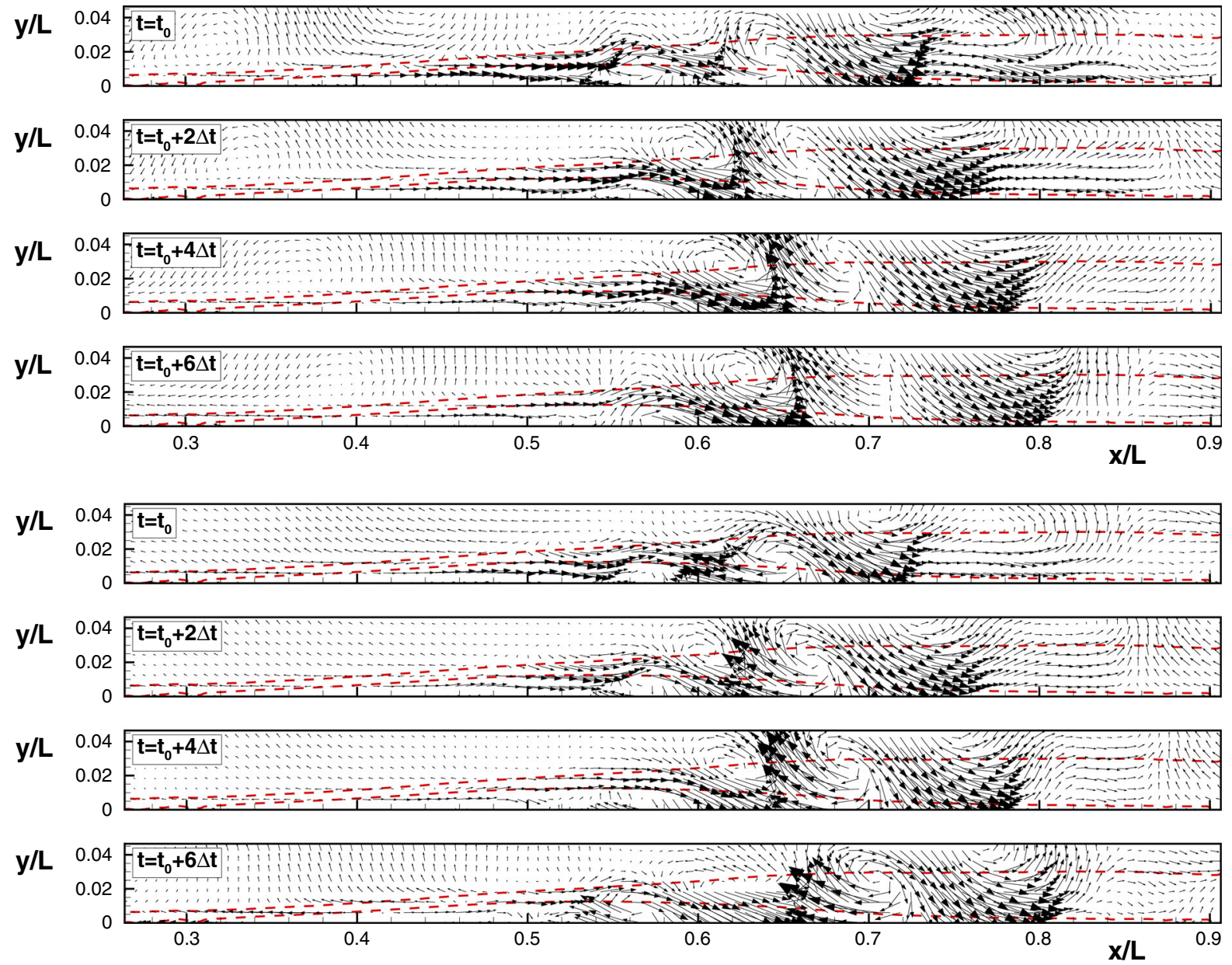

Fig. 20 Sequence of perturbation velocity maps for separation induced transition case reconstructed using 10 (top) NE-POD and (bottom) classic POD modes. Mean flow structure is highlighted by iso-contour lines of the mean velocity (red lines). (Color figure online)

related fluctuations, as well as their possible correlation.

Figure 20 shows two sequences of NE-POD (top plots) and POD (bottom plots) filtered snapshots for the case of separation induced transition. Both loworder reconstructions clearly highlight the propagation of large scale $\mathrm{K}-\mathrm{H}$ vortices from the maximum displacement of the laminar separation bubble $(x / L>0.6)$. In the first NE-POD filtered snapshot (top plot) a clock-wise rotating vortex seems to form at $x / L=0.56$, which is shown to move forward in the successive images. It should be noted that the same pattern is not clearly observable in the corresponding snapshot obtained by reconstruction from the classic POD modes. This is due to the higher energy characterizing the velocity fluctuations downstream of the bubble maximum displacement, which prevents the characterization of the formation process of new $\mathrm{K}-\mathrm{H}$ rolls. Additionally, the low-order filtered field obtained by means of NE-POD modes better highlights the occurrence of stramwise fluctuations in the separated shear layer for $x / L<0.56$ (compare the first and second snapshots of both low-order representations) due to BL streaks, as well as the propagation of large scale vortices in the free-stream region. These latter structures, which are well captured by the NEPOD filtered field, are not instead observable in any of low-order POD representation. The present results therefore suggest that the characterization of freestream vortices and of streaky structures, which propagate in the separated shear layer, as well as their link to the formation of $\mathrm{K}-\mathrm{H}$ rolls, may be performed 
looking at low-order models obtained by means of the NE-POD method here discussed.

\section{Conclusions}

In the present work, different variants of the classic POD method, including temporal and spatial normalization matrices and weighted scalar product, have been applied to both numerical and experimental data. The capability of these procedures to capture turbulent events embedded in low energy spatio-temporal regions has been discussed and highlighted.

For the specific flows at hand, the normalization of the energy of each snapshot has been proved to introduce only negligible variation with respect to the classic POD procedure. This is due to the similar energy level of the snapshots, different to what occurs in highly phase dependent flow fields tested in the literature. In the conditions examined in this work, the normalization only acts "scaling" the data set. Significant different modal representation characterizes instead the SI-POD method, where the spatial weight matrix acts normalizing the rows of the snapshot matrix, thus better highlighting oscillation events embedded in spatial region with low kinetic energy. This procedure has been proved to better emphasize free-stream events for the experimental test cases considered. However, it almost completely hides the occurrence of energetic events sparse in time (like streaky structures in the by-pass transition process). Such events are not recovered applying both spatial and temporal normalization to the data matrix since the spatial one dominates, being characterized by a significantly larger rms of its diagonal entries. The adaptation of a weighted projection into the NE-POD method provides, in the case of separation induced and by-pass transition beneath free-stream turbulence, the greatest capability to identify oscillations events occurring in low energetic spatial region as well as sparse in time. In the case of bypass transition, the occurrence of boundary layer streaks and less energetic free-stream vortices has been clearly highlighted by the NE-POD method. The Fourier decomposition of the modes allowed isolating the low-frequency streaky structures from the high-frequency free-stream vortices. Interestingly, $Q_{2}$ events related to streak breakdown are characterized by the same frequency contributions characterizing free-stream fluctuations.
In the case of separated flow transition, the NE-POD procedure further highlights velocity fluctuations in the fore part of the bubble. Low frequency contributions to the modes well isolate streaky-like structures upstream of the bubble maximum displacement, while higher frequencies have been found to be mostly related to the $\mathrm{K}-\mathrm{H}$ rolls, originating downstream of this position. Moreover, high frequency contributions to the modes also highlight free-stream structures at the edge of the separating boundary layer, similar to those observed in the case of bypass transition.

Low-order reconstruction of the velocity field obtained by means of POD and NE-POD modes in case of by-pass and separation induced transition were presented. NE-POD based low-order models have been shown to better highlight lower energy events such as free-stream vortices and ordered coherent structures preceding the breakup process of both streaky structures and $\mathrm{K}-\mathrm{H}$ rolls in the attached and separated boundary layer, respectively.

Funding Open access funding provided by Università degli Studi di Genova within the CRUI-CARE Agreement.

\section{Declarations}

Conflict of interest The authors declare that they have no conflict of interest.

Open Access This article is licensed under a Creative Commons Attribution 4.0 International License, which permits use, sharing, adaptation, distribution and reproduction in any medium or format, as long as you give appropriate credit to the original author(s) and the source, provide a link to the Creative Commons licence, and indicate if changes were made. The images or other third party material in this article are included in the article's Creative Commons licence, unless indicated otherwise in a credit line to the material. If material is not included in the article's Creative Commons licence and your intended use is not permitted by statutory regulation or exceeds the permitted use, you will need to obtain permission directly from the copyright holder. To view a copy of this licence, visit http://creativecommons.org/licenses/by/4.0/.

\section{References}

1. Antoranz A, Ianiro A, Flores O, García-Villalba M (2018) Extended proper orthogonal decomposition of non-homogeneous thermal fields in a turbulent pipe flow. Int $\mathrm{J}$ Heat Mass Transfer 118:1264-1275

2. Arndt REA, Long DF, Glauser MN (1997) The proper orthogonal decomposition of pressure fluctuations surrounding a turbulent jet. J Fluid Mech 340:1-33 
3. Borée J (2003) Extended proper orthogonal decomposition: a tool to analyse correlated events in turbulent flows. Exp Fluids 35(2):188-192

4. Bourgeois JA, Noack BR, Martinuzzi RJ (2013) Generalized phase average with applications to sensor-based flow estimation of the wall-mounted square cylinder wake. J Fluid Mech 736:316-350

5. Brunton SL, Proctor JL, Kutz JN (2016) Discovering governing equations from data by sparse identification of nonlinear dynamical systems. In: Proceedings of the national academy of sciences, p 201517384

6. Citriniti JH, George WK (2000) Reconstruction of the global velocity field in the axisymmetric mixing layer utilizing the proper orthogonal decomposition. J Fluid Mech 418:137-166

7. Cosadia I, Borée J, Dumont P (2007) Coupling time-resolved piv flow-fields and phase-invariant proper orthogonal decomposition for the description of the parameters space in a transparent diesel engine. Exp Fluids 43(2):357-370

8. Discetti S, Raiola M, Ianiro A (2018) Estimation of timeresolved turbulent fields through correlation of non-timeresolved field measurements and time-resolved point measurements. Exp Ther Fluid Sci 93:119-130

9. Diwan SS, Ramesh ON (2009) On the origin of the inflectional instability of a laminar separation bubble. J Fluid Mech 629:263-298

10. Fogleman M, Lumley J, Rempfer D, Haworth D (2002) Analysis of tumble breakdown in ic engines using phaseinvariant POD modes

11. Fogleman M, Lumley J, Rempfer D, Haworth D (2004) Application of the proper orthogonal decomposition to datasets of internal combustion engine flows. J Turbul 5(23):1-3

12. Glauser MN, George WK (1987) Orthogonal decomposition of the axisymmetric jet mixing layer including azimuthal dependence. In: Advances in turbulence, Springer, pp 357-366

13. Glauser MN, George WK (1992) Application of multipoint measurements for flow characterization. Exp Ther Fluid Sci 5(5):617-632

14. Hosseini Z, Martinuzzi RJ, Noack BR (2015) Sensor-based estimation of the velocity in the wake of a low-aspect-ratio pyramid. Exp Fluids 56(1):13

15. Istvan MS, Yarusevych S (2018) Effects of free-stream turbulence intensity on transition in a laminar separation bubble formed over an airfoil. Exp Fluids 59(3):52

16. Iudiciani P, Duwig C, Hosseini S, Szasz R, Fuchs L, Gutmark E, Lantz A, Collin R, Aldén M (2010) Proper orthogonal decomposition for experimental investigation of swirling flame instabilities. In: 48th AIAA aerospace sciences meeting including the new horizons forum and aerospace exposition, p 584

17. Jacob RG, Durbin PA (2001) Simulations of bypass transition. J Fluid Mech 428:185-212

18. Kutz JN, Brunton SL, Brunton BW, Proctor JL (2016) Dynamic mode decomposition: data-driven modeling of complex systems, vol 149. SIAM

19. Lengani D, Simoni D, Ubaldi M, Zunino P (2014) POD analysis of the unsteady behavior of a laminar separation bubble. Exp Ther Fluid Sci 58:70-79

20. Lengani D, Simoni D, Ubaldi M, Zunino P, Bertini F (2017) Analysis of the Reynolds stress component production in a laminar separation bubble. Int $\mathrm{J}$ Heat Fluid Flow 64:112-119

21. Lengani D, Simoni D, Nilberto A, Ubaldi M, Zunino P, Bertini F (2018a) Synchronization of multi-plane measurement data by means of pod: application to unsteady boundary layer transition. Exp Fluids 59(12):184

22. Lengani D, Simoni D, Pichler R, Sandberg R, Michelassi V, Bertini F (2018b) Identification and quantification of losses in a LPT cascade by POD applied to LES data. Int J Heat Fluid Flows 70:28-40

23. Liu Z, Adrian RJ, Hanratty TJ (2001) Large-scale modes of turbulent channel flow: transport and structure. J Fluid Mech 448:53-80

24. Lumley JL (1970) Stochastic tools in turbulence. Applied mathematics and mechanics, vol 12

25. Marxen O, Henningson DS (2011) The effect of smallamplitude convective disturbances on the size and bursting of a laminar separation bubble. J Fluid Mech 671:1-33

26. Marxen O, Rist U (2010) Mean flow deformation in a laminar separation bubble: separation and stability characteristics. J Fluid Mech 660:37-54

27. Marxen O, Lang M, Rist U (2013) Vortex formation and vortex breakup in a laminar separation bubble. J Fluid Mech 728:58-90

28. Mendez M, Balabane M, Buchlin JM (2019) Multi-scale proper orthogonal decomposition of complex fluid flows. J Fluid Mech 870:988-1036

29. Michelis T, Yarusevych S, Kotsonis M (2018) On the origin of spanwise vortex deformations in laminar separation bubbles. J Fluid Mech 841:81-108

30. Nolan K, Zaki T (2013) Conditional sampling of transitional boundary layers in pressure gradients. J Fluid Mech 728:306-339

31. Perrin R, Cid E, Cazin S, Sevrain A, Braza M, Moradei F, Harran G (2007) Phase-averaged measurements of the turbulence properties in the near wake of a circular cylinder at high Reynolds number by 2C-PIV and 3C-PIV. Exp Fluids 42:93-109

32. Sarmast S, Dadfar R, Mikkelsen RF, Schlatter P, Ivanell S, Sørensen JN, Henningson DS (2014) Mutual inductance instability of the tip vortices behind a wind turbine. J Fluid Mech 755:705-731

33. Satta F, Simoni D, Ubaldi M, Zunino P, Bertini F, Spano E (2007) Velocity and turbulence measurements in a separating boundary layer with and without passive flow control. Proc Inst Mech Eng Part A J Power Energy 221(6):815-818

34. Shi LL, Liu YZ, Wan JJ (2010) Influence of wall proximity on characteristics of wake behind a square cylinder: PIV measurements and POD analysis. Exp Therm Fluid Sci 34:28-36

35. Sieber M, Paschereit CO, Oberleithner K (2016) Spectral proper orthogonal decomposition. J Fluid Mech 792:798-828

36. Simoni D, Lengani D, Guida R (2016a) A wavelet-based intermittency detection technique from PIV investigations in transitional boundary layers. Exp Fluids 57(9):145

37. Simoni D, Lengani D, Ubaldi M, Zunino P, Guida R (2016b) Turbulence production, dissipation and length scales in laminar separation bubbles. In: ETMM11

38. Simoni D, Lengani D, Ubaldi M, Zunino P, Dellacasagrande M (2017) Inspection of the dynamic properties of laminar 
separation bubbles: free-stream turbulence intensity effects for different Reynolds numbers. Exp Fluids 58(6):66

39. Sirovich L (1987) Turbulence and the dynamics of coherent structures. part I-III. Q Appl Math 45:561-590

40. Taira K, Colonius T (2007) The immersed boundary method: a projection approach. J Comput Phys 225(2):2118-2137

41. Tinney CE, Glauser MN, Ukeiley LS (2008) Low-dimensional characteristics of a transonic jet. part 1. proper orthogonal decomposition. J Fluid Mech 612:107-141

42. Voisine M, Thomas L, Borée J, Rey P (2011) Spatio-temporal structure and cycle to cycle variations of an in-cylinder tumbling flow. Exp Fluids 50(5):1393-1407

43. Yang Z, Voke PR (2001) Large-eddy simulation of boundary-layer separation and transition at a change of surface curvature. J Fluid Mech 439:305-333
44. Yarusevych S, Kawall JG, Sullivan PE (2008) Separatedshear-layer development on an airfoil at low Reynolds numbers. AIAA J 46(12):3060-3069

45. Zaki T (2013) From streaks to spots and on to turbulence: exploring the dynamics of boundary layer transition. Flow Turbul Combust 91:451-473

46. Zaki TA, Saha S (2009) On shear sheltering and the structure of vortical modes in single-and two-fluid boundary layers. J Fluid Mech 626:111-147

Publisher's Note Springer Nature remains neutral with regard to jurisdictional claims in published maps and institutional affiliations. 